\title{
Article
}

\section{Boundedness of commutators on herz-morry-hardy spaces with variable exponent}

\author{
Omer Abdalrhman ${ }^{1,2 *}$, Afif Abdalmonem ${ }^{1,3}$ and Shuangping Tao ${ }^{1}$ \\ 1 College of Mathematics and Statistics, Northwest Normal University, Lanzhou, Gansu, P.R. China.; \\ afeefy86@gmail.com(A.A); taosp@nwnu.edu.cn(S.T). \\ 2 College of Education, Shendi University, Shendi, River Nile State, Sudan. \\ 3 Faculty of Science, University of Dalanj, Dalanj, South kordofan, Sudan. \\ * Correspondence: humoora@gmail.com
}

Received: 18 October 2018; Accepted: 11 March 2019; Published: 16 April 2019.

Abstract: In this paper, we obtain the boundedness of commutators generated by the Calderón-Zygmund operator, BMO functions and Lipschitz function on Herz-Morrey-Hardy spaces with variable exponent $H M K_{p(\cdot), \lambda}^{\alpha(\cdot), q}\left(\mathbb{R}^{n}\right)$.

Keywords: Simpson's inequality, strongly $(s, m)$-convex functions, numerical quadrature rule, Hölder inequality.

MSC: 26A51, 26D15, 26D10, 41A55.

\section{Introduction}

$\mathbf{S}$

uppose $\mathbb{S}^{n-1},(n \geq 2)$ denote the unit sphere in $\mathbb{R}^{n}$ equipped with the normalized Lebesgue measure $\mathrm{d} \sigma=\mathrm{d}\left(\sigma^{\prime}\right)$. Let $\Omega$ be homogeneous function of degree zero and satisfies

$$
\int_{\mathbb{S}^{n-1}} \Omega\left(x^{\prime}\right) \mathrm{d} \sigma\left(x^{\prime}\right), \text { where } x^{\prime}=x /|x|(x \neq 0) .
$$

The Calderón-Zygmund singular integral operator $T_{\Omega}$ is defined as

$$
T_{\Omega} h(x)=\text { p.v. } \int_{\mathbb{R}^{n}} \frac{\Omega(x-y)}{|x-y|^{n}} h(y) \mathrm{d} y .
$$

Now, we recall the definitions of the corresponding commutators of the Calderón-Zygmund singular integral operator. Suppose that $b \in \mathrm{BMO}\left(\mathbb{R}^{n}\right)$, the commutators $\left[b, T_{\Omega}\right]$ generated by $b$ and $T$ is defined as

$$
\left[b, T_{\Omega}\right] h(x)=\text { p.v. } \int_{\mathbb{R}^{n}} \frac{\Omega(x-y)}{|x-y|^{n}}[b(x)-b(y)] h(y) \mathrm{d} y .
$$

These operators were firstly introduced by Calderón and Zygmund in [1,2], in which they proved that these operator are bounded on $L^{p}$, where $0<p<1$. Coifman et al. [3] showed that if $\Omega \in \dot{\Lambda}_{\gamma}\left(\mathbb{S}^{n-1}\right)$ where $\gamma \in(0,1)$ and $b \in \operatorname{BMO}\left(\mathbb{R}^{n}\right)$, then $\left[b, T_{\Omega}\right]$ is bounded on $L^{p}$. In 2011, Lu Ding and Yan [4] proved that $T_{\Omega}$ and the commutator $\left[b, T_{\Omega}\right]$ are bounded on weighted $\left(L^{p}\left(\mathbb{R}^{n}\right), L^{q}\left(\mathbb{R}^{n}\right)\right)$.

In last 30 years, the function spaces with variable exponent have attracted researchers since the paper [5] appeared in 1991, see, for example [6-10] and their references. Recently, Jingshi Xu and Xiaodi Yang [11] studied the Herz-Morrey-Hardy spaces with variable exponent and their applications.

Motivated by [11-13], our main purpose of this paper is to study some boundedness for commutators of Calderón-Zygmund operators on Herz-Morrey-Hardy space with two variable exponents. The main tools are properties of variable exponent, BMO function and Lipschitz function.

Definition 1. Let $\Omega \subset \mathbb{R}^{n}$ be a subset of $\mathbb{R}^{n}$ with the Lebesgue measure $>0$. For a measurable function $p(\cdot): \Omega \rightarrow[1, \infty)$, the variable Lebesgue space is defined as

$$
L^{p(\cdot)}(\Omega):=\left\{h \text { is measurable on } \Omega: \rho_{p}(h)<\infty\right\},
$$


where

$$
\rho_{p}(h):=\int_{\Omega}\left(\frac{|h(x)|}{\mu}\right)^{p(x)} d x<\infty \text { for some constat } \mu>0 .
$$

The set $L^{p(\cdot)}(\Omega)$ is a quasi Banach space with following Luxemburg-Nakano norm

$$
\|h\|_{L^{p(\cdot)}}:=\inf \left\{\mu>0: \rho_{p}\left(\mu^{-1} h\right) \leq 1\right\} .
$$

The space $L_{\mathrm{loc}}^{p(\cdot)}(\Omega)$ is defined as

$$
L_{\text {loc }}^{p(\cdot)}(\Omega):=\left\{h: h \chi_{k} \in L^{p(\cdot)}\left(\mathbb{R}^{n}\right) \text { for any compact subset } K \subset \Omega\right\} .
$$

Suppose $\mathcal{P}(\Omega)$ represents the set of all function $p: \Omega \rightarrow[1, \infty)$. Assume that $p_{-}=\operatorname{essinf}_{x \in \Omega} p(x)$ and $p_{+}=\operatorname{ess}_{\sup _{x \in \Omega}} p(x)$. Set $p_{-}>1, p_{+}<\infty$ and $p(\cdot), p^{\prime}(\cdot)$ are conjugate exponent function defined by $1 / p(\cdot)+1 / p^{\prime}(\cdot)=1$. Let $\mathcal{B}(\Omega)$ be the set of $p(\cdot) \in \mathcal{P}(\Omega)$ satisfying that the maximal function is bounded on $L^{p(\cdot)}$.

Definition 2. (see[11]). Let $p(\cdot) \in \mathcal{P}\left(\mathbb{R}^{n}\right), 0<q<\infty, 0 \leq \lambda<\infty, \alpha(\cdot) \in L^{\infty}\left(\mathbb{R}^{n}\right)$ and $N>n+1$. The homogeneous Herz-Morrey-Hardy spaces $\operatorname{HMK}_{p(\cdot), \lambda}^{\alpha(\cdot), q}\left(\mathbb{R}^{n}\right)$ and nonhomogeneous Herz-Morrey-Hardy spaces $H M K_{p(\cdot), \lambda}^{\alpha(\cdot), q}\left(\mathbb{R}^{n}\right)$ are defined as

$$
\begin{aligned}
& H M \dot{K}_{p(\cdot), \lambda}^{\alpha(\cdot), q}\left(\mathbb{R}^{n}\right)=\left\{h \in \mathcal{S}^{\prime}\left(\mathbb{R}^{n}\right):\|h\|_{H M \dot{K}_{p(\cdot), \lambda}^{\alpha(\cdot), q}\left(\mathbb{R}^{n}\right)}:=\left\|G_{N} h\right\|_{M \dot{K}_{p(\cdot), \lambda}^{\alpha(\cdot), q}\left(\mathbb{R}^{n}\right)}<\infty\right\}, \\
& H M K_{p(\cdot), \lambda}^{\alpha(\cdot), q}\left(\mathbb{R}^{n}\right)=\left\{h \in \mathcal{S}^{\prime}\left(\mathbb{R}^{n}\right):\|h\|_{H M K_{p(\cdot), \lambda}^{\alpha(\cdot), q}\left(\mathbb{R}^{n}\right)}:=\left\|G_{N} h\right\|_{M \dot{K}_{p(\cdot), \lambda}^{\alpha(\cdot), q}\left(\mathbb{R}^{n}\right)}<\infty\right\} .
\end{aligned}
$$

respectively.

\section{Preliminaries and Lemmas}

Proposition 3. (see[14]). Given a function $p(\cdot): \mathbb{R}^{n} \rightarrow[1, \infty)$. If $p(\cdot) \in \mathcal{P}\left(\mathbb{R}^{n}\right)$ satisfies

$$
|p(x)-p(y)| \leq \frac{-C}{\log (|x-y|)} ; \quad|x-y| \leq 1 / 2,
$$

and

$$
|p(x)-p(y)| \leq \frac{C}{\log (e+|x|)} ; \quad|y| \geq|x|
$$

then, $p(\cdot) \in \mathfrak{B}\left(\mathbb{R}^{n}\right)$.

Lemma 4. (see[5]). (Generalized Hölder Inequality) Given $p(\cdot), p_{1}(\cdot), p_{2}(\cdot) \in \mathcal{P}\left(\mathbb{R}^{n}\right)$.

1. For every $h \in L^{p_{1}(\cdot)}\left(\mathbb{R}^{n}\right)$ and $g \in L^{p_{2}(\cdot)}\left(\mathbb{R}^{n}\right)$, we have

$$
\int_{\mathbb{R}^{n}}|h(x) g(x)| d x \leq C\|h\|_{L^{p(\cdot)}\left(\mathbb{R}^{n}\right)}\|g\|_{L^{p^{\prime}(\cdot)}\left(\mathbb{R}^{n}\right)^{\prime}}
$$

where $C_{p}=1+\frac{1}{p_{-}}-\frac{1}{p_{+}}$.

2. For every $h \in L^{p_{1}(\cdot)}\left(\mathbb{R}^{n}\right)$, and $g \in L^{p_{2}(\cdot)}\left(\mathbb{R}^{n}\right)$, when $\frac{1}{p(\cdot)}=\frac{1}{p_{2}(\cdot)}+\frac{1}{p_{1}(\cdot)}$, we have

$$
\|h(x) g(x)\|_{L^{p(\cdot)}\left(\mathbb{R}^{n}\right)} \leq C\|g(x)\|_{L^{p_{2}\left(\mathbb{R}^{n}\right)}}\|h(x)\|_{L^{p_{1}(\cdot)}\left(\mathbb{R}^{n}\right)^{\prime}}
$$

where $C_{p_{1}, p_{2}}=\left[1+\frac{1}{p_{1-}}-\frac{1}{p_{1+}}\right]^{\frac{1}{p_{-}}}$. 
Lemma 5. (see $[15,16]$ ). Given $p(\cdot) \in \mathcal{B}\left(\mathbb{R}^{n}\right)$. If there exist positive constants $C, \delta_{1}$ and $\delta_{2}$ such that $\delta_{1}, \delta_{2}<1$, then for all balls $B \subset \mathbb{R}^{n}$ and all measurable subset $R \subset B$, we have

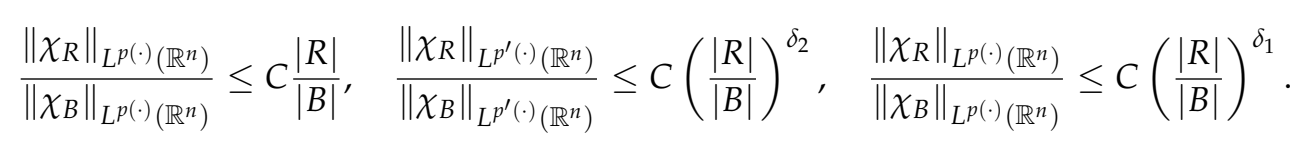

Lemma 6. (see[17]). If $p(\cdot) \in \mathcal{B}\left(\mathbb{R}^{n}\right)$, then there exists a constant $C>0$ such that for any ball $B$ in $\mathbb{R}^{n}$, we have

$$
\frac{1}{|B|}\left\|\chi_{B}\right\|_{L^{p(\cdot)}\left(\mathbb{R}^{n}\right)}\left\|\chi_{B}\right\|_{L^{p^{\prime}(\cdot)}\left(\mathbb{R}^{n}\right)} \leq C
$$

Now, the BMO function and BMO norm are defined as

$$
\begin{aligned}
\operatorname{BMO}\left(\mathbb{R}^{n}\right) & :=\left\{b \in L_{l o c}^{1}\left(\mathbb{R}^{n}\right):\|b\|_{\mathrm{BMO}\left(\mathbb{R}^{n}\right)}<0\right\}, \\
\|b\|_{\mathrm{BMO}\left(\mathbb{R}^{n}\right)} & :=\sup _{Q}|Q|^{-1} \int_{Q}\left|b(x)-b_{Q}\right| d x .
\end{aligned}
$$

respectively.

Lemma 7. (see[8]). Given $p(\cdot) \in \mathcal{B}\left(\mathbb{R}^{n}\right), b \in \mathrm{BMO}\left(\mathbb{R}^{n}\right)$. If $i, j \in \mathbb{Z}$ with $i<j$, then we have

1. $C^{-1}\|b\|_{\mathrm{BMO}\left(\mathbb{R}^{n}\right)} \leq \sup _{B} \frac{1}{\left\|\chi_{B}\right\|_{L^{p(\cdot)}\left(\mathbb{R}^{n}\right)}}\left\|\left(b-b_{B}\right) \chi_{B}\right\|_{L^{p(\cdot)}\left(\mathbb{R}^{n}\right)} \leq C\|b\|_{\mathrm{BMO}\left(\mathbb{R}^{n}\right)}$.

2. $\left\|\left(b-b_{B_{i}}\right) \chi_{B_{j}}\right\|_{L^{q(\cdot)}\left(\mathbb{R}^{n}\right)} \leq C(j-i)\|b\|_{\mathrm{BMO}\left(\mathbb{R}^{n}\right)}\left\|\chi_{B_{j}}\right\|_{L^{q(\cdot)}\left(\mathbb{R}^{n}\right)}$.

Lemma 8. (see[18]). Suppose that $p(\cdot) \in \mathcal{P}\left(\mathbb{R}^{n}\right), q \in[0, \infty)$ and $\lambda \in[0, \infty)$. If $\alpha(\cdot)$ is log-Hölder continuous both at origin and at infinity, then

$$
\begin{gathered}
\|h\|_{M \dot{K}_{p(\cdot), \lambda}^{\alpha(\cdot),\left(\mathbb{R}^{n}\right)}} \approx \max \left\{\sup _{L \leq 0, L \in \mathbb{Z}} 2^{-L \lambda}\left(\sum_{k=-\infty}^{L} 2^{k \alpha(0) q}\left\|h \chi_{k}\right\|_{L^{p(\cdot)}\left(\mathbb{R}^{n}\right)}^{q}\right)^{1 / q},\right. \\
\left.\sup _{L>0, L \in \mathbb{Z}}\left[2^{-L \lambda}\left(\sum_{k=-\infty}^{-1} 2^{k \alpha(0) q}\left\|h \chi_{k}\right\|_{L^{p(\cdot)}\left(\mathbb{R}^{n}\right)}^{q}\right)^{1 / q}+2^{-L \lambda}\left(\sum_{k=0}^{L} 2^{k \alpha_{\infty} q}\left\|h \chi_{k}\right\|_{L^{p(\cdot)}\left(\mathbb{R}^{n}\right)}^{q}\right)^{1 / q}\right]\right\} .
\end{gathered}
$$

Lemma 9. (see[4]). Let $\Omega$ satisfies $L^{r}$-Dini condition $r \in[1, \infty)$. If there exist constants $C>0$ and $R>0$ such that $|y|<R / 2$, then for every $x \in \mathbb{R}^{n}$, we have

$$
\left(\int_{R<|x|<2 R}\left|\frac{\Omega(x-y)}{|x-y|^{n}}-\frac{\Omega(x)}{|x|^{n}}\right|^{r} d x\right)^{\frac{1}{r}} \leq C R^{\left(\frac{n}{r}-n\right)}\left\{\frac{|y|}{R}+\int_{|y| / 2 R<\delta<|y| / R} \frac{w_{r}(\delta)}{\delta} d \delta\right\} .
$$

Lemma 10. (see[11]). Suppose that $p(\cdot) \in \mathcal{B}\left(\mathbb{R}^{n}\right), q \in[0, \infty)$ and $\lambda \in[0, \infty)$. Let $\alpha(\cdot)$ is log-Hölder continuous both at origin and at infinity. If $2 \lambda \leq \alpha(\cdot), n \delta_{2} \leq \alpha(0), \alpha<\infty$ and $\delta_{2}$ as defined in Lemma 5 . Then $h \in H M \dot{K}_{p(\cdot), \lambda}^{\alpha(\cdot), q}\left(\mathbb{R}^{n}\right) \quad\left(\right.$ or $\left.H M K_{p(\cdot), \lambda}^{\alpha(\cdot), q}\left(\mathbb{R}^{n}\right)\right)$ if and only if $h=\sum_{k=-\infty}^{\infty} \lambda_{k} g_{k}\left(\right.$ or $\left.\sum_{k=0}^{\infty} \lambda_{k} g_{k}\right)$, in the sense of $\mathcal{S}^{\prime}\left(\mathbb{R}^{n}\right)$, where each $g_{k}$ be a central $(\alpha(\cdot), p(\cdot))$-atom (or central $(\alpha(\cdot), p(\cdot))$-atom of restricted type) with support contained in $B_{k}$ and $\sup _{L \in \mathbb{Z}} 2^{-L \lambda} \sum_{k=-\infty}^{L}\left|\lambda_{k}\right|^{q}<\infty$ or $\left(\sup _{L \in \mathbb{Z}} 2^{-L \lambda} \sum_{k=0}^{L}\left|\lambda_{k}\right|^{q}<\infty\right)$.

Also,

$\|h\|_{H M \dot{K}_{p(\cdot), \lambda}^{\alpha(\cdot), q}} \approx \inf _{L \in \mathbb{Z}} \sup ^{L \lambda}\left(\sum_{k=-\infty}^{L}\left|\lambda_{k}\right|^{q}\right)^{1 / q}\left(\right.$ or $\left.\|h\|_{H M K_{p(\cdot), \lambda}^{\alpha(\cdot), q}} \approx \inf _{L \in \mathbb{Z}} 2^{L \lambda}\left(\sum_{k=0}^{L}\left|\lambda_{k}\right|^{q}\right)^{1 / q}\right)$, where infimum is taken over all above decomposition of $h$.

Lemma 11. (see [19]). Let $p(\cdot) \in \mathcal{P}(\Omega)$ and $h: \Omega \times \Omega \rightarrow \mathbb{R}$ is a measurable function (with respect to product measure) such that, for almost every $y \in \Omega, h(\cdot, y) \in L^{p(\cdot)}(\Omega)$. Then

$$
\left\|\int_{\Omega} h(\cdot, y) d y\right\|_{L^{p(\cdot)}(\Omega)} \leq C \int_{\Omega}\|h(\cdot, y)\|_{L^{p(\cdot)}(\Omega)} d y .
$$


Lemma 12. (see[19]). Suppose $p(\cdot) \in \mathcal{P}\left(\mathbb{R}^{n}\right)$ satisfies conditions (4) and (5) of Proposition 3, then for any ball (or cube) $Q \subset \mathbb{R}^{n}$, we have

$$
\left\|\chi_{Q}\right\|_{L^{p(\cdot)}\left(\mathbb{R}^{n}\right)} \approx \begin{cases}|Q|^{\frac{1}{p(x)}}, & \text { if }|Q| \leq 2^{n} \\ |Q|^{\frac{1}{p(\infty)}}, & \text { if }|Q| \geq 1\end{cases}
$$

where $p(\infty)=\lim _{x \rightarrow \infty} p(x)$.

\section{Main Results}

In this section, we formulate and prove the main results of this paper.

Theorem 13. Let $p(\cdot) \in \mathcal{B}\left(\mathbb{R}^{n}\right)$ and $\Omega \in L^{r}\left(\mathbb{S}^{n-1}\right)\left(r>p^{+}\right)$satisfies

$$
\int_{0}^{1} \frac{w_{r}(\delta)}{\delta^{1+\beta}} d \delta<\infty, \quad 0<\beta \leq 1
$$

Suppose that $0<q<\infty, 0 \leq \lambda<\infty$ and $\alpha(\cdot) \in L^{\infty}\left(\mathbb{R}^{n}\right)$ satisfies conditions (4) and (5) of Proposition 3 . If $2 \lambda \leq$ $\alpha(\cdot), n \delta_{2} \leq \alpha(0), \alpha_{\infty}<\beta+n \delta_{2}$, then $T_{\Omega}$ is bounded from $H M \dot{K}_{p(\cdot), \lambda}^{\alpha(\cdot), q}$ or $\left(H M K_{p(\cdot), \lambda}^{\alpha(\cdot), q}\right)$ to $M \dot{K}_{p(\cdot), \lambda}^{\alpha(\cdot), q}$ or $\left(M K_{p(\cdot), \lambda}^{\alpha(\cdot), q}\right)$.

Proof. It suffices to prove for $H M \dot{K}_{p(\cdot), \lambda}^{\alpha(\cdot), q}$. Assume that $h \in H M \dot{K}_{p(\cdot), \lambda}^{\alpha(\cdot), q}$, then by Lemma 10, $h=$ $\sum_{j=-\infty}^{\infty} \lambda_{j} g_{j}$ converges in $\mathcal{S}^{\prime}\left(\mathbb{R}^{n}\right)$, where $\|h\|_{H M \dot{K}_{p(\cdot), \lambda}^{\alpha(\cdot), q}} \approx \inf _{L \in \mathbb{Z}} 2^{L \lambda}\left(\sum_{j=-\infty}^{L}\left|\lambda_{j}\right|^{q}\right)^{1 / q}$, and $g_{j}$ is a dyadic central $(\alpha(\cdot), p(\cdot))$-atom with support contained in $B_{j}$. For simplicity, we take $Y=\sup _{L \in \mathbb{Z}} 2^{L \lambda} \sum_{j=-\infty}^{L}\left|\lambda_{j}\right|^{q}$. By virtue of Lemma 8, we have

$$
\begin{aligned}
& \left\|T_{\Omega}(h)\right\|_{M \dot{K}_{p(\cdot), \lambda}^{\alpha(\cdot), q}\left(\mathbb{R}^{n}\right)} \approx \max \left\{\sup _{L \leq 0, L \in \mathbb{Z}} 2^{-L \lambda}\left(\sum_{k=-\infty}^{L} 2^{k \alpha(0) q}\left\|T_{\Omega}(h) \chi_{k}\right\|_{L^{p(\cdot)}\left(\mathbb{R}^{n}\right)}^{q}\right)^{1 / q},\right. \\
& \left.\sup _{L>0, L \in \mathbb{Z}}\left[2^{-L \lambda}\left(\sum_{k=-\infty}^{-1} 2^{k \alpha(0) q}\left\|T_{\Omega}(h) \chi_{k}\right\|_{L^{p(\cdot)}\left(\mathbb{R}^{n}\right)}^{q}\right)^{1 / q}+2^{-L \lambda}\left(\sum_{k=0}^{L} 2^{k \alpha_{\infty} q}\left\|T_{\Omega}(h) \chi_{k}\right\|_{L^{p(\cdot)}\left(\mathbb{R}^{n}\right)}^{q}\right)^{1 / q}\right]\right\} \\
& \approx \max \{E, F+G\} .
\end{aligned}
$$

Let

$$
\begin{aligned}
E & =\sup _{L \leq 0, L \in \mathbb{Z}} 2^{-L \lambda q} \sum_{k=-\infty}^{L} 2^{k \alpha(0) q}\left\|T_{\Omega}(h) \chi_{k}\right\|_{L^{p(\cdot)}\left(\mathbb{R}^{n}\right)^{\prime}}^{q}, \\
F & =\sum_{k=-\infty}^{-1} 2^{k \alpha(0) q}\left\|T_{\Omega}(h) \chi_{k}\right\|_{L^{p(\cdot)}\left(\mathbb{R}^{n}\right)^{\prime}}^{q} \\
G & =\sup _{L>0, L \in \mathbb{Z}} 2^{-L \lambda q} \sum_{k=0}^{L} 2^{k \alpha_{\infty} q}\left\|T_{\Omega}(h) \chi_{k}\right\|_{L^{p(\cdot)}\left(\mathbb{R}^{n}\right)}^{q} .
\end{aligned}
$$

To finish our proof, we only need to show that there exists a constant $C>0$, such that $E, F, G \leq C Y$.

First we prove that $E \leq C Y$.

$$
\begin{aligned}
E= & \left.\sup _{L \leq 0, L \in \mathbb{Z}} 2^{-L \lambda q} \sum_{k=-\infty}^{L} 2^{k \alpha(0) q}\left\|T_{\Omega}(h) \chi_{k}\right\|_{L^{p(\cdot)}}^{q} \mathbb{R}^{n}\right) \\
& \left.+\sup _{L \leq 0, L \in \mathbb{Z}} 2^{-L \lambda q} \sum_{k=-L \in \mathbb{Z}}^{L} 2^{-L \lambda q} 2_{k=-\infty}^{L \alpha(0) q}\left(\sum_{j=k}^{\infty}\left|\lambda_{j}\right| \| T_{\Omega} g_{j}\right) \chi_{k} \|_{L^{p(\cdot)}\left(\mathbb{R}^{n}\right)}\right)^{q} \\
& \operatorname{sul}^{k(0) q}\left(\sum_{j=-\infty}^{k-1}\left|\lambda_{j}\right|\left\|T_{\Omega}\left(g_{j}\right) \chi_{k}\right\|_{L^{p(\cdot)}\left(\mathbb{R}^{n}\right)}\right)^{q}:=E_{1}+E_{2} .
\end{aligned}
$$

By the $\left(L^{p(\cdot)}\left(\mathbb{R}^{n}\right), L^{p(\cdot)}\left(\mathbb{R}^{n}\right)\right)$-boundedness of the $T_{\Omega}$ (see[13]), we get

$$
\left\|T_{\Omega}\left(g_{j}\right) \chi_{k}\right\|_{L^{p(\cdot)}\left(\mathbb{R}^{n}\right)} \leq\left\|g_{j}\right\|_{L^{p(\cdot)}\left(\mathbb{R}^{n}\right)} \leq\left|B_{j}\right|^{-\alpha_{j} / n}=2^{-j \alpha_{j}} .
$$


Therefore, when $0<q \leq 1$, we obtain

$$
\begin{aligned}
& E_{1}=\sup _{L \leq 0, L \in \mathbb{Z}} 2^{-L \lambda q} \sum_{k=-\infty}^{L} 2^{k \alpha(0) q}\left(\sum_{j=k}^{\infty}\left|\lambda_{j}\right|\left\|T_{\Omega}\left(g_{j}\right) \chi_{k}\right\|_{L^{p(\cdot)}\left(\mathbb{R}^{n}\right)}\right)^{q} \\
& \leq C \sup _{L \leq 0, L \in \mathbb{Z}} 2^{-L \lambda q} \sum_{k=-\infty}^{L} 2^{k \alpha(0) q}\left(\sum_{j=k}^{\infty}\left|\lambda_{j}\right| 2^{-j \alpha_{j}}\right)^{q} \\
& \leq C \sup _{L \leq 0, L \in \mathbb{Z}} 2^{-L \lambda q} \sum_{k=-\infty}^{L} 2^{k \alpha(0) q}\left[\left(\sum_{j=k}^{-1}\left|\lambda_{j}\right| 2^{-j \alpha(0)}+\sum_{j=0}^{\infty}\left|\lambda_{j}\right| 2^{-j \alpha_{\infty}}\right)^{q}\right] \\
& \leq C\left[\sup _{L \leq 0, L \in \mathbb{Z}} 2^{-L \lambda q} \sum_{k=-\infty}^{L} \sum_{j=k}^{-1}\left|\lambda_{j}\right|^{q} 2^{(k-j) \alpha(0) q}+\sup _{L \leq 0, L \in \mathbb{Z}} 2^{-L \lambda q}\left(\sum_{k=-\infty}^{L} 2^{k \alpha(0)} \sum_{j=0}^{\infty}\left|\lambda_{j}\right| 2^{-j \alpha_{\infty}}\right)^{q}\right] \\
& \leq C\left[\sup _{L \leq 0, L \in \mathbb{Z}} 2^{-L \lambda q} \sum_{j=-\infty}^{-1}\left|\lambda_{j}\right|^{q} \sum_{k=-\infty}^{j} 2^{(k-j) \alpha(0) q}+\sup _{L \leq 0, L \in \mathbb{Z}} \sum_{j=0}^{\infty} 2^{-j \lambda q}\left|\lambda_{j}\right|^{q} 2^{\left(\lambda-\alpha_{\infty}\right) j q} 2^{-L \lambda q} \sum_{k=\infty}^{L} 2^{k \alpha(0) q}\right] \\
& \leq C\left[\sup _{L \leq 0, L \in \mathbb{Z}} 2^{-L \lambda q} \sum_{j=-\infty}^{L}\left|\lambda_{j}\right|^{q}+\sup _{L \leq 0, L \in \mathbb{Z}} 2^{-L \lambda q} \sum_{j=L}^{-1}\left|\lambda_{j}\right|^{q} \sum_{k=-\infty}^{j} 2^{(k-j) \alpha(0) q}\right. \\
& \left.+\mathrm{Y} \sup _{L \leq 0, L \in \mathbb{Z}} \sum_{j=0}^{\infty} 2^{\left(\lambda-\alpha_{\infty}\right) j q} \sum_{k=\infty}^{L} 2^{[k \alpha(0)-L \lambda] q}\right] \\
& \leq C\left[\mathrm{Y}+\sup _{L \leq 0, L \in \mathbb{Z}} \sum_{j=L}^{-1} 2^{-j \lambda q}\left|\lambda_{j}\right|^{q} 2^{(j-L) \lambda q} \sum_{k=-\infty}^{j} 2^{(k-j) \alpha(0) q}+\mathrm{Y}\right] \\
& \leq C\left[\mathrm{Y}+\mathrm{Y} \sup _{L \leq 0, L \in \mathbb{Z}} \sum_{j=L}^{-1} 2^{(j-L) \lambda q} \sum_{k=-\infty}^{j} 2^{(k-j) \alpha(0) q}+\mathrm{Y}\right] \leq C \mathrm{Y} .
\end{aligned}
$$

when $1<q<\infty$, and $1 / q+1 / q^{\prime}=1$, we have

$$
\begin{aligned}
E_{1}= & \sup _{L \leq 0, L \in \mathbb{Z}} 2^{-L \lambda q} \sum_{k=-\infty}^{L} 2^{k \alpha(0) q}\left(\sum_{j=k}^{\infty}\left|\lambda_{j}\right|\left\|T_{\Omega}\left(g_{j}\right) \chi_{k}\right\|_{L^{p(\cdot)}\left(\mathbb{R}^{n}\right)}\right)^{q} \\
\leq & C \sup _{L \leq 0, L \in \mathbb{Z}} 2^{-L \lambda q} \sum_{k=-\infty}^{L} 2^{k \alpha(0) q}\left(\sum_{j=k}^{\infty}\left|\lambda_{j}\right| 2^{-j \alpha_{j}}\right)^{q} \\
\leq & C\left[\sup _{L \leq 0, L \in \mathbb{Z}} 2^{-L \lambda q} \sum_{k=-\infty}^{L}\left(\sum_{j=k}^{-1}\left|\lambda_{j}\right| 2^{(k-j) \alpha(0)}\right)^{q}+\sup _{L \leq 0, L \in \mathbb{Z}} 2^{-L \lambda q} \sum_{k=-\infty}^{L} 2^{k \alpha(0) q}\left(\sum_{j=0}^{\infty}\left|\lambda_{j}\right| 2^{-j \alpha_{\infty}}\right)^{q}\right] \\
\leq & C\left[\sup _{L \leq 0, L \in \mathbb{Z}} 2^{-L \lambda q} \sum_{k=-\infty}^{L}\left(\sum_{j=k}^{-1}\left|\lambda_{j}\right|^{q} 2^{(k-j) \alpha(0) \frac{q}{2}}\right) \times\left(\sum_{j=k}^{-1} 2^{(k-j) \alpha(0) \frac{q^{\prime}}{2}}\right)^{\frac{q}{q^{\prime}}}\right. \\
& \left.+\sup _{L \leq 0, L \in \mathbb{Z}} 2^{-L \lambda q} \sum_{k=-\infty}^{L} 2^{k \alpha(0) q}\left(\sum_{j=0}^{\infty}\left|\lambda_{j}\right|^{q} 2^{-j \alpha_{\infty} \frac{q}{2}}\right) \times\left(\sum_{j=0}^{\infty} 2^{-j \alpha_{\infty} \frac{q^{\prime}}{2}}\right)^{\frac{q}{q^{\prime}}}\right] \\
\leq & C\left[\sup _{L \leq 0, L \in \mathbb{Z}} 2^{-L \lambda q} \sum_{k=-\infty}^{L} \sum_{j=k}^{-1}\left|\lambda_{j}\right|^{q} 2^{(k-j) \alpha(0) \frac{q}{2}}+\sup _{L \leq 0, L \in \mathbb{Z}} 2^{-L \lambda q} \sum_{k=-\infty}^{L} 2^{k \alpha(0) q} \sum_{j=0}^{\infty}\left|\lambda_{j}\right|^{q} 2^{-j \alpha_{\infty} \frac{q}{2}}\right] \\
\leq & C\left[\sup _{L \leq 0, L \in \mathbb{Z}} 2^{-L \lambda q} \sum_{j=-\infty}^{-1}\left|\lambda_{j}\right|^{q} \sum_{k=\infty}^{j} 2^{(k-j) \alpha(0) \frac{q}{2}}+\sup _{L \leq 0, L \in \mathbb{Z}} \sum_{j=0}^{\infty} 2^{-j \lambda q}\left|\lambda_{j}\right|^{q} 2^{\left(\lambda-\frac{\alpha \infty}{2}\right) j q} 2^{-L \lambda q} \sum_{k=-\infty}^{L} 2^{k \alpha(0) q}\right] \\
\leq & C\left[\sup _{L \leq 0, L \in \mathbb{Z}} 2^{-L \lambda q} \sum_{j=-\infty}^{L}\left|\lambda_{j}\right|^{q}+\sup _{L \leq 0, L \in \mathbb{Z}} 2^{-L \lambda q} \sum_{j=L}^{-1}\left|\lambda_{j}\right|^{q} \sum_{k=\infty}^{j} 2^{(k-j) \alpha(0) \frac{q}{2}}\right. \\
& \left.+\mathrm{Y} \sup _{L \leq 0, L \in \mathbb{Z}} \sum_{j=0}^{\infty} 2^{\left(\lambda-\frac{\alpha \infty}{2}\right) j q} 2^{-L \lambda q} \sum_{k=-\infty}^{L} 2^{k \alpha(0) q}\right]
\end{aligned}
$$




$$
\begin{aligned}
& \leq C\left[\mathrm{Y}+\sup _{L \leq 0, L \in \mathbb{Z}} \sum_{j=L}^{-1} 2^{-j \lambda q}\left|\lambda_{j}\right|^{q} 2^{(j-L) \lambda q} \sum_{k=\infty}^{j} 2^{(k-j) \alpha(0) \frac{q}{2}}+\mathrm{Y}\right] \\
& \leq C\left[\mathrm{Y}+\mathrm{Y} \sup _{L \leq 0, L \in \mathbb{Z}} \sum_{j=L}^{-1} 2^{(j-L) \lambda q} \sum_{k=\infty}^{j} 2^{(k-j) \alpha(0) \frac{q}{2}}+\mathrm{Y}\right] \\
& \leq \mathrm{CY} .
\end{aligned}
$$

Now, we prove that $E_{2} \leq C Y$. Note that if $x \in A_{k}$ for each $k \in \mathbb{Z}, y \in A_{j}$ and $j \leq k-1$. Let $\tilde{p}(\cdot)>$ 1 and $1 / p(\cdot)=1 / \tilde{p}(\cdot)+1 / r$. Since $r>p^{+}$, so by Lemma 4 and Lemma 11 , we get

$$
\begin{aligned}
\left\|T_{\Omega}\left(g_{j}\right) \chi_{k}\right\|_{L^{p(\cdot)}\left(\mathbb{R}^{n}\right)} & \leq \int_{B_{j}}\left\|\frac{\Omega(\cdot-y)}{|\cdot-y|^{n}}-\frac{\Omega(\cdot)}{|\cdot|^{n}}\left|\chi_{k} \|_{L^{p(\cdot)}\left(\mathbb{R}^{n}\right)}\right| g_{j}(y) \mid d y\right. \\
& \leq \int_{B_{j}}\left\|\frac{\Omega(\cdot-y)}{|\cdot-y|^{n}}-\frac{\Omega(\cdot)}{|\cdot|^{n}}\right\|_{L^{r}\left(\mathbb{R}^{n}\right)}\left\|\chi_{k}\right\|_{L^{\tilde{p}(\cdot)}\left(\mathbb{R}^{n}\right)}\left|g_{j}(y)\right| d y .
\end{aligned}
$$

Using Lemma 9, we get

$$
\begin{aligned}
\left\|\frac{\Omega(\cdot-y)}{|\cdot-y|^{n}}-\frac{\Omega(\cdot)}{|\cdot|^{n}}\right\|_{L^{r}\left(\mathbb{R}^{n}\right)} & \leq C 2^{(k-1)\left(\frac{n}{r}-n\right)}\left\{\frac{|y|}{2^{k-1}}+\int_{|y| / 2^{k}}^{|y| / 2^{k-1}} \frac{w_{r}(\delta)}{\delta} d \delta\right\} \\
& \leq C 2^{(k-1)\left(\frac{n}{r}-n\right)}\left(2^{j-k}+2^{(j-k) \beta} \int_{0}^{1} \frac{w_{r}(\delta)}{\delta^{1+\beta}} d \delta\right) \\
& \leq C 2^{(k-1)\left(\frac{n}{r}-n\right)} 2^{(j-k) \beta} .
\end{aligned}
$$

By Lemma 12, we obtain

$$
\begin{aligned}
\left\|T_{\Omega}\left(g_{j}\right) \chi_{k}\right\|_{L^{p(\cdot)}\left(\mathbb{R}^{n}\right)} & \leq\left\|\left|\frac{\Omega(\cdot-y)}{|\cdot-y|^{n}}-\frac{\Omega(\cdot)}{|\cdot|^{n}}\right|\right\|_{L^{r}\left(\mathbb{R}^{n}\right)}\left\|\chi_{k}\right\|_{L^{\tilde{p}(\cdot)}\left(\mathbb{R}^{n}\right)} \int_{B_{j}}\left|g_{j}(y)\right| d y \\
& \leq C\|\| \frac{\Omega(\cdot-y)}{|\cdot-y|^{n}}-\frac{\Omega(\cdot)}{|\cdot|^{n}} \mid\left\|_{L^{r}\left(\mathbb{R}^{n}\right)}\left(\left\|\chi_{B_{k}}\right\|_{L^{p(\cdot)}\left(\mathbb{R}^{n}\right)}\left|B_{k}\right|^{\frac{1}{r}}\right)\right\| g_{j} \|_{L^{p(\cdot)}\left(\mathbb{R}^{n}\right)} .
\end{aligned}
$$

From (8) and Lemmas 4-6, we get

$$
\begin{aligned}
\left\|T_{\Omega}\left(g_{j}\right) \chi_{k}\right\|_{L^{p(\cdot)\left(\mathbb{R}^{n}\right)}} & \leq C 2^{-n k+(j-k) \beta}\left(\left\|\chi_{B_{k}}\right\|_{L^{\left.p^{(} \cdot\right)}\left(\mathbb{R}^{n}\right)}^{-1}\left|B_{k}\right|\right)\left\|g_{j}\right\|_{L^{p(\cdot)}\left(\mathbb{R}^{n}\right)}\left\|\chi_{B_{j}}\right\|_{L^{p^{\prime}(\cdot)\left(\mathbb{R}^{n}\right)}} \\
& \leq C 2^{(j-k) \beta}\left(\frac{\left\|\chi_{B_{j}}\right\|_{L^{p^{\prime}(\cdot)}\left(\mathbb{R}^{n}\right)}}{\left\|\chi_{B_{k}}\right\|_{L^{p^{\prime}(\cdot)\left(\mathbb{R}^{n}\right)}}}\right)\left\|g_{j}\right\|_{L^{p(\cdot)\left(\mathbb{R}^{n}\right)}} \\
& \leq C 2^{(j-k)\left(\beta+n \delta_{2}\right)-j \alpha_{j}}
\end{aligned}
$$

So, when $0<q \leq 1$, we obtain

$$
\begin{aligned}
E_{2} & =\sup _{L \leq 0, L \in \mathbb{Z}} 2^{-L \lambda q} \sum_{k=-\infty}^{L} 2^{k \alpha(0) q}\left(\sum_{j=-\infty}^{k-1}\left|\lambda_{j}\right|\left\|T_{\Omega}\left(g_{j}\right) \chi_{k}\right\|_{L^{p(\cdot)}\left(\mathbb{R}^{n}\right)}\right)^{q} \\
& \left.\leq C \sup _{L \leq 0, L \in \mathbb{Z}} 2^{-L \lambda q} \sum_{k=-\infty}^{L} 2^{k \alpha(0) q}\left(\sum_{j=-\infty}^{k-1}\left|\lambda_{j}\right|\right) 2^{(j-k)\left(\beta+n \delta_{2}\right)-j \alpha(0)}\right)^{q} \\
& \leq C \sup _{L \leq 0, L \in \mathbb{Z}} 2^{-L \lambda q} \sum_{k=-\infty}^{L}\left(\sum_{j=-\infty}^{k-1}\left|\lambda_{j}\right| 2^{\left[(j-k)\left(\beta+n \delta_{2}\right)-\alpha(0)\right]}\right)^{q} \\
& \leq C \sup _{L \leq 0, L \in \mathbb{Z}} 2^{-L \lambda q} \sum_{j=-\infty}^{L}\left|\lambda_{j}\right|^{q}\left(\sum_{k=j+1}^{-1} 2^{\left[(j-k)\left(\beta+n \delta_{2}\right)-\alpha(0)\right]}\right)^{q} \\
& \leq C Y .
\end{aligned}
$$


When $0<q<\infty$, and $1 / q+1 / q^{\prime}=1$, by $n \delta_{2} \leq \alpha(0)<\beta+n \delta_{2}$ and Hölder's inequality, we have

$$
\begin{aligned}
E_{2}= & \sup _{L \leq 0, L \in \mathbb{Z}} 2^{-L \lambda q} \sum_{k=-\infty}^{L} 2^{k \alpha(0) q}\left(\sum_{j=-\infty}^{k-1}\left|\lambda_{j}\right|\left\|T_{\Omega}\left(g_{j}\right) \chi_{k}\right\|_{L^{p(\cdot)}\left(\mathbb{R}^{n}\right)}\right)^{q} \\
\leq & C \sup _{L \leq 0, L \in \mathbb{Z}} 2^{-L \lambda q} \sum_{k=-\infty}^{L}\left(\sum_{j=-\infty}^{k-1}\left|\lambda_{j}\right|^{q} 2^{\left[(j-k)\left(\beta+n \delta_{2}\right)-\alpha(0)\right] \frac{q}{2}}\right) \\
& \times\left(\sum_{j=-\infty}^{k-1} 2^{\left[(j-k)\left(\beta+n \delta_{2}\right)-\alpha(0)\right] \frac{q^{\prime}}{2}}\right)^{\frac{q}{q^{\prime}}} \\
\leq & C \sup _{L \leq 0, L \in \mathbb{Z}} 2^{-L \lambda q} \sum_{j=-\infty}^{L}\left|\lambda_{j}\right|^{q} \sum_{k=j+1}^{-1} 2^{\left[(j-k)\left(\beta+n \delta_{2}\right)-\alpha(0)\right] \frac{q}{2}} \\
\leq & C Y .
\end{aligned}
$$

Next we prove that $F \leq C Y$.

$$
\begin{aligned}
F= & \sum_{k=-\infty}^{-1} 2^{k \alpha(0) q}\left\|T_{\Omega}(h) \chi_{k}\right\|_{L^{p(\cdot)}\left(\mathbb{R}^{n}\right)}^{q} \leq \sum_{k=-\infty}^{-1} 2^{k \alpha(0) q}\left(\sum_{j=k}^{\infty}\left|\lambda_{j}\right|\left\|T_{\Omega}\left(g_{j}\right) \chi_{k}\right\|_{L^{p(\cdot)}\left(\mathbb{R}^{n}\right)}\right)^{q} \\
& +\sum_{k=-\infty}^{-1} 2^{k \alpha(0) q}\left(\sum_{j=-\infty}^{k-1}\left|\lambda_{j}\right|\left\|T_{\Omega}\left(g_{j}\right) \chi_{k}\right\|_{L^{p(\cdot)}\left(\mathbb{R}^{n}\right)}\right)^{q} \\
:= & F_{1}+F_{2} .
\end{aligned}
$$

Since $0<q \leq 1$, we get

$$
\begin{aligned}
F_{1} & =\sum_{k=-\infty}^{-1} 2^{k \alpha(0) q}\left(\sum_{j=k}^{\infty}\left|\lambda_{j}\right|\left\|T_{\Omega}\left(g_{j}\right) \chi_{k}\right\|_{L^{p(\cdot)}\left(\mathbb{R}^{n}\right)}\right)^{q} \leq C \sum_{k=-\infty}^{-1} 2^{k \alpha(0) q}\left(\sum_{j=k}^{\infty}\left|\lambda_{j}\right| 2^{-j \alpha_{j}}\right)^{q} \\
& \leq C \sum_{k=-\infty}^{-1} 2^{k \alpha(0) q}\left[\sum_{j=k}^{-1}\left|\lambda_{j}\right|^{q} 2^{-j \alpha(0) q}+\sum_{j=0}^{\infty}\left|\lambda_{j}\right|^{q} 2^{-j \alpha_{\infty} q}\right] \\
& \leq C\left[\sum_{k=-\infty}^{-1} \sum_{j=k}^{-1}\left|\lambda_{j}\right|^{q} 2^{(k-j) \alpha(0) q}+\sum_{k=-\infty}^{-1} 2^{k \alpha(0) q} \sum_{j=0}^{\infty}\left|\lambda_{j}\right|^{q} 2^{-j \alpha_{\infty} q}\right] \\
& \leq C\left[\sum_{j=-\infty}^{-1}\left|\lambda_{j}\right|^{q} \sum_{k=-\infty}^{j} 2^{(k-j) \alpha(0) q}+\sum_{k=-\infty}^{-1} 2^{k \alpha(0) q} \sum_{j=0}^{\infty} 2^{-j \lambda q} \sum_{l=0}^{j}\left|\lambda_{l}\right|^{q} 2^{\left(\lambda-\alpha_{\infty}\right) j q}\right] \\
& \leq C\left[\mathrm{Y}+\mathrm{Y} \sum_{k=-\infty}^{-1} 2^{k \alpha(0) q} \sum_{j=0}^{\infty} 2^{\left(\lambda-\alpha_{\infty}\right) j q}\right] \\
& \leq C Y .
\end{aligned}
$$

when $0<q<\infty$ and $1 / q+1 / q^{\prime}=1$, we deduce

$$
\begin{aligned}
F_{1}= & \sum_{k=-\infty}^{-1} 2^{k \alpha(0) q}\left(\sum_{j=k}^{\infty}\left|\lambda_{j}\right||| T_{\Omega}\left(g_{j}\right) \chi_{k} \|_{L^{p(\cdot)}\left(\mathbb{R}^{n}\right)}\right)^{q} \leq C \sum_{k=-\infty}^{-1} 2^{k \alpha(0) q}\left(\sum_{j=k}^{\infty}\left|\lambda_{j}\right| 2^{-j \alpha_{j}}\right)^{q} \\
\leq & C\left[\sum_{k=-\infty}^{-1}\left(\sum_{j=k}^{-1}\left|\lambda_{j}\right| 2^{(k-j) \alpha(0)}\right)^{q}+\sum_{k=-\infty}^{-1} 2^{k \alpha(0) q}\left(\sum_{j=0}^{\infty}\left|\lambda_{j}\right| 2^{-j \alpha_{\infty}}\right)^{q}\right] \\
\leq & C\left[\sum_{k=-\infty}^{-1}\left(\sum_{j=k}^{-1}\left|\lambda_{j}\right|^{q} 2^{(k-j) \alpha(0) \frac{q}{2}}\right) \times\left(\sum_{j=k}^{-1} 2^{(k-j) \alpha(0) \frac{q^{\prime}}{2}}\right)^{\frac{q}{q^{\prime}}}\right. \\
& \left.+\sum_{k=-\infty}^{-1} 2^{k \alpha(0) q}\left(\sum_{j=0}^{\infty}\left|\lambda_{j}\right|^{q} 2^{-j \alpha_{\infty} \frac{q}{2}}\right) \times\left(\sum_{j=0}^{\infty} 2^{-j \alpha_{\infty} \frac{q^{\prime}}{2}}\right)^{\frac{q}{q^{\prime}}}\right]
\end{aligned}
$$




$$
\begin{aligned}
& \leq C\left[\sum_{k=-\infty}^{-1} \sum_{j=k}^{-1}\left|\lambda_{j}\right|^{q} 2^{(k-j) \alpha(0) \frac{q}{2}}+\sum_{k=-\infty}^{-1} 2^{k \alpha(0) q} \sum_{j=0}^{\infty}\left|\lambda_{j}\right|^{q} 2^{-j \alpha_{\infty} \frac{q}{2}}\right] \\
& \leq C\left[\sum_{j=-\infty}^{-1}\left|\lambda_{j}\right|^{q} \sum_{k=\infty}^{j} 2^{(k-j) \alpha(0) \frac{q}{2}}+\sum_{j=0}^{\infty} 2^{-j \lambda q} \sum_{l=-\infty}^{j}\left|\lambda_{l}\right|^{q} 2^{\left(\lambda-\frac{\alpha \infty}{2}\right) j q} \sum_{k=-\infty}^{-1} 2^{k \alpha(0) q}\right] \\
& \leq C\left[Y \sum_{k=\infty}^{j} 2^{(k-j) \alpha(0) \frac{q}{2}}+Y \sum_{j=0}^{\infty} 2^{\left(\lambda-\frac{\alpha \infty}{2}\right) j q} \sum_{k=-\infty}^{-1} 2^{k \alpha(0) q}\right] \\
& \leq C Y .
\end{aligned}
$$

Now we prove that $F_{2} \leq C Y$. When $0<q \leq 1$, from (11) and $n \delta_{2} \leq \alpha(0)<\beta+n \delta_{2}$, we have

$$
\begin{aligned}
F_{2}= & \sum_{k=-\infty}^{-1} 2^{k \alpha(0) q}\left(\sum_{j=-\infty}^{k-1}\left|\lambda_{j}\right|\left\|T_{\Omega}\left(g_{j}\right) \chi_{k}\right\|_{L^{p(\cdot)}\left(\mathbb{R}^{n}\right)}\right)^{q} \leq C \sum_{k=-\infty}^{-1} 2^{k \alpha(0) q}\left(\sum_{j=-\infty}^{k-1}\left|\lambda_{j}\right| 2^{(j-k)\left(\beta+n \delta_{2}\right)-j \alpha(0)}\right)^{q} \\
& \leq C \sum_{k=-\infty}^{-1}\left(\sum_{j=-\infty}^{k-1}\left|\lambda_{j}\right| 2^{\left[(j-k)\left(\beta+n \delta_{2}\right)-\alpha(0)\right]}\right)^{q} \leq C \sum_{j=-\infty}^{-1}\left|\lambda_{j}\right|^{q}\left(\sum_{k=j+1}^{-1} 2^{\left[(j-k)\left(\beta+n \delta_{2}\right)-\alpha(0)\right]}\right)^{q} \\
& \leq C Y .
\end{aligned}
$$

when $0<q \leq \infty$, and $1 / q+1 / q^{\prime}=1$, by $n \delta_{2} \leq \alpha(0)<\beta+n \delta_{2}$ and Hölder's inequality, we obtain

$$
\begin{aligned}
F_{2} & =\sum_{k=-\infty}^{-1} 2^{k \alpha(0) q}\left(\sum_{j=-\infty}^{k-1}\left|\lambda_{j}\right|\left\|T_{\Omega}\left(g_{j}\right) \chi_{k}\right\|_{L^{p(\cdot)}\left(\mathbb{R}^{n}\right)}\right)^{q} \\
& \leq C \sum_{k=-\infty}^{-1}\left(\sum_{j=-\infty}^{k-1}\left|\lambda_{j}\right|^{q} 2^{\left[(j-k)\left(\beta+n \delta_{2}\right)-\alpha(0)\right] \frac{q}{2}}\right) \times\left(\sum_{j=-\infty}^{k-1} 2^{\left[(j-k)\left(\beta+n \delta_{2}\right)-\alpha(0)\right] \frac{q^{\prime}}{2}}\right)^{\frac{q}{q^{\prime}}} \\
& \leq C \sum_{j=-\infty}^{-1}\left|\lambda_{j}\right|^{q} \sum_{k=j+1}^{-1} 2^{\left[(j-k)\left(\beta+n \delta_{2}\right)-\alpha(0)\right] \frac{q}{2}} \\
& \leq C Y .
\end{aligned}
$$

Finally we prove that $G \leq C Y$.

$$
\begin{aligned}
G & =\sup _{L>0, L \in \mathbb{Z}} 2^{-L \lambda q} \sum_{k=0}^{L} 2^{k \alpha_{\infty} q}\left\|T_{\Omega}(h) \chi_{k}\right\|_{L^{p(\cdot)}\left(\mathbb{R}^{n}\right)}^{q} \leq \sup _{L>0, L \in \mathbb{Z}} 2^{-L \lambda q} \sum_{k=0}^{L} 2^{k \alpha_{\infty} q}\left(\sum_{j=k}^{\infty}\left|\lambda_{j}\right|\left\|T_{\Omega}\left(g_{j}\right) \chi_{k}\right\|_{L^{p(\cdot)}\left(\mathbb{R}^{n}\right)}\right)^{q} \\
& +\sup _{L>0, L \in \mathbb{Z}} 2^{-L \lambda q} \sum_{k=0}^{L} 2^{k \alpha_{\infty} q}\left(\sum_{j=-\infty}^{k-1}\left|\lambda_{j}\right|\left\|T_{\Omega}\left(g_{j}\right) \chi_{k}\right\|_{L^{p(\cdot)}\left(\mathbb{R}^{n}\right)}\right)^{q} \\
& :=G_{1}+G_{2} .
\end{aligned}
$$

When $0<q \leq 1$, by the boundedness of the commutator $[b, T]$ in $L^{p(\cdot)}\left(\mathbb{R}^{n}\right)$, we obtain

$$
\begin{aligned}
G_{1}= & \sup _{L>0, L \in \mathbb{Z}} 2^{-L \lambda q} \sum_{k=0}^{L} 2^{k \alpha_{\infty} q}\left(\sum_{j=k}^{\infty}\left|\lambda_{j}\right|\left\|T_{\Omega}\left(g_{j}\right) \chi_{k}\right\|_{L^{p(\cdot)}\left(\mathbb{R}^{n}\right)}\right)^{q} \leq C \sup _{L>0, L \in \mathbb{Z}} 2^{-L \lambda q} \sum_{k=0}^{L} 2^{k \alpha_{\infty} q}\left(\sum_{j=k}^{\infty}\left|\lambda_{j}\right| 2^{-j \alpha_{j}}\right)^{q} \\
& \leq C \sup _{L>0, L \in \mathbb{Z}} 2^{-L \lambda q} \sum_{k=0}^{L} 2^{k \alpha_{\infty} q}\left(\sum_{j=k}^{L}\left|\lambda_{j}\right|^{q} 2^{-j \alpha_{\infty} q}+\sum_{j=L}^{\infty}\left|\lambda_{j}\right|^{q} 2^{-j \alpha_{\infty} q}\right) \\
& \leq C\left(\sup _{L>0, L \in \mathbb{Z}} 2^{-L \lambda q} \sum_{j=0}^{L}\left|\lambda_{j}\right|^{q} \sum_{k=0}^{j} 2^{(k-j) \alpha_{\infty} q}+\sup _{L>0, L \in \mathbb{Z}} 2^{-L \lambda q} \sum_{j=L}^{\infty}\left|\lambda_{j}\right|^{q} \sum_{k=0}^{L} 2^{(k-j) \alpha_{\infty} q}\right) \\
& \leq C\left(\sup _{L>0, L \in \mathbb{Z}} 2^{-L \lambda q} \sum_{j=0}^{L}\left|\lambda_{j}\right|^{q} \sum_{k=0}^{j} 2^{(k-j) \alpha_{\infty} q}+\sup _{L>0, L \in \mathbb{Z}} \sum_{j=L}^{\infty} 2^{j \lambda q-L \lambda q} 2^{-j \lambda q} \sum_{l=-\infty}^{j}\left|\lambda_{l}\right|^{q} \sum_{k=0}^{L} 2^{(k-j) \alpha_{\infty} q}\right) \\
& \leq C\left(\mathrm{Y}+\mathrm{Y} \sup _{L>0, L \in \mathbb{Z}} \sum_{j=L}^{\infty} 2^{(j-L) \lambda q} 2^{(L-j) \alpha_{\infty} q}\right) \leq C\left(\mathrm{Y}+\mathrm{Y} \sup _{L>0, L \in \mathbb{Z}} \sum_{j=L}^{\infty} 2^{\left[(j-L)\left(\lambda-\alpha_{\infty}\right)\right] q}\right) \leq C Y .
\end{aligned}
$$


When $0<q<\infty$, by using Hölder's inequality, we have

$$
\begin{aligned}
& G_{1}=\sup _{L>0, L \in \mathbb{Z}} 2^{-L \lambda q} \sum_{k=0}^{L} 2^{k \alpha_{\infty} q}\left(\sum_{j=k}^{\infty}\left|\lambda_{j}\right|\left\|T_{\Omega}\left(g_{j}\right) \chi_{k}\right\|_{L^{p(\cdot)}\left(\mathbb{R}^{n}\right)}\right)^{q} \\
& \leq C \sup _{L>0, L \in \mathbb{Z}} 2^{-L \lambda q} \sum_{k=0}^{L} 2^{k \alpha_{\infty} q}\left(\sum_{j=k}^{\infty}\left|\lambda_{j}\right|^{q}\left|B_{j}\right|^{-j \alpha_{j} \frac{q}{2 n}}\right) \times\left(\sum_{j=k}^{\infty}\left|B_{j}\right|^{-j \alpha_{j} \frac{q^{\prime}}{2 n}}\right)^{\frac{q}{q^{\prime}}} \\
& \leq C \sup _{L>0, L \in \mathbb{Z}} 2^{-L \lambda q} \sum_{k=0}^{L}\left(\sum_{j=k}^{\infty}\left|\lambda_{j}\right|^{q} 2^{(k-j) \alpha_{\infty} \frac{q}{2}}\right) \\
& \leq C\left[\sup _{L>0, L \in \mathbb{Z}} 2^{-L \lambda q} \sum_{j=0}^{L}\left|\lambda_{j}\right|^{q} \sum_{k=0}^{j} 2^{(k-j) \alpha_{\infty} \frac{q}{2}}+\sup _{L>0, L \in \mathbb{Z}} 2^{-L \lambda q} \sum_{j=L}^{\infty}\left|\lambda_{j}\right|^{q} \sum_{k=0}^{L} 2^{(k-j) \alpha_{\infty} \frac{q}{2}}\right] \\
& \leq C\left[\sup _{L>0, L \in \mathbb{Z}} 2^{-L \lambda q} \sum_{j=0}^{L}\left|\lambda_{j}\right|^{q}+\sup _{L>0, L \in \mathbb{Z}} \sum_{j=L}^{\infty} 2^{(j-L) \lambda q} 2^{-j \lambda q} \sum_{l=-\infty}^{j}\left|\lambda_{l}\right|^{q} \sum_{k=0}^{L} 2^{(k-j) \alpha_{\infty} \frac{q}{2}}\right] \\
& \leq C\left[Y+Y \sup _{L>0, L \in \mathbb{Z}} \sum_{j=L}^{\infty} 2^{(j-L) \lambda q} 2^{(L-j) \frac{\alpha \infty}{2} q}\right] \\
& \leq C\left[\mathrm{Y}+\mathrm{Y} \sup _{L>0, L \in \mathbb{Z}} \sum_{j=L}^{\infty} 2^{(j-L)\left(\lambda-\frac{\alpha \infty}{2}\right) q}\right] \\
& \leq \mathrm{CY} \text {. }
\end{aligned}
$$

For $G_{2} \leq C Y$, when $0<q<\infty$, and $1 / q+1 / q^{\prime}=1$, from (11), $n \delta_{2} \leq \alpha(0), \alpha_{\infty}<\beta+n \delta_{2}$ and applying Hölder's inequality, we have

$$
\begin{aligned}
& G_{2}=\sup _{L>0, L \in \mathbb{Z}} 2^{-L \lambda q} \sum_{k=0}^{L} 2^{k \alpha_{\infty} q}\left(\sum_{j=-\infty}^{k-1}\left|\lambda_{j}\right|\left\|T_{\Omega}\left(g_{j}\right) \chi_{k}\right\|_{L^{p(\cdot)}\left(\mathbb{R}^{n}\right)}\right)^{q} \\
& \leq C \sup _{L>0, L \in \mathbb{Z}} 2^{-L \lambda q} \sum_{k=0}^{L} 2^{k \alpha_{\infty} q}\left(\sum_{j=-\infty}^{k-1}\left|\lambda_{j}\right| 2^{(j-k)\left(\beta+n \delta_{2}\right)-j \alpha_{j}}\right)^{q} \\
& \leq C \sup _{L>0, L \in \mathbb{Z}} 2^{-L \lambda q} \sum_{k=0}^{L} 2^{k \alpha_{\infty} q}\left(\sum_{j=-\infty}^{-1}\left|\lambda_{j}\right| 2^{(j-k)\left(\beta+n \delta_{2}\right)-j \alpha(0)}\right)^{q} \\
& +C \sup _{L>0, L \in \mathbb{Z}} 2^{-L \lambda q} \sum_{k=0}^{L} 2^{k \alpha_{\infty} q}\left(\sum_{j=0}^{k-1}\left|\lambda_{j}\right| 2^{(j-k)\left(\beta+n \delta_{2}\right)-j \alpha_{\infty}}\right)^{q} \\
& \leq C \sup _{L>0, L \in \mathbb{Z}} 2^{-L \lambda q} \sum_{k=0}^{L} 2^{\left[\alpha_{\infty}-\left(\beta+n \delta_{2}\right)\right] k q}\left(\sum_{j=-\infty}^{-1}\left|\lambda_{j}\right| 2^{\left[\left(\beta+n \delta_{2}\right)-\alpha(0)\right] j}\right)^{q} \\
& +C \sup _{L>0, L \in \mathbb{Z}} 2^{-L \lambda q} \sum_{k=0}^{L}\left(\sum_{j=0}^{k-1}\left|\lambda_{j}\right| 2^{(j-k)\left[\left(\beta+n \delta_{2}\right)-\alpha_{\infty}\right]}\right)^{q} \\
& \leq C \sup _{L>0, L \in \mathbb{Z}} 2^{-L \lambda q} \sum_{k=0}^{L} 2^{\left[\alpha_{\infty}-\left(\beta+n \delta_{2}\right)\right] k q}\left(\sum_{j=-\infty}^{-1}\left|\lambda_{j}\right| 2^{j \frac{\left[\left(\beta+n \delta_{2}\right)-\alpha(0)\right]}{2}}\right)^{q} \times\left(\sum_{j=-\infty}^{-1}(k-j)^{q^{\prime}} 2^{j\left[\left(\beta+n \delta_{2}\right)-\alpha(0)\right] \frac{q^{\prime}}{2}}\right)^{\frac{q}{q^{\prime}}} \\
& +C \sup _{L>0, L \in \mathbb{Z}} 2^{-L \lambda q} \sum_{k=0}^{L}\left(\sum_{j=0}^{k-1}\left|\lambda_{j}\right| 2^{(j-k) \frac{\left[\left(\beta+n \delta_{2}\right)-\alpha_{\infty}\right]}{2}}\right)^{q} \times\left(\sum_{j=0}^{k-1}(k-j)^{q^{\prime}} 2^{(j-k)\left[\left(\beta+n \delta_{2}\right)-\alpha_{\infty}\right] \frac{q^{\prime}}{2}}\right)^{\frac{q}{q^{\prime}}} \\
& \leq C \sup _{L>0, L \in \mathbb{Z}} 2^{-L \lambda q} \sum_{j=-\infty}^{-1}\left|\lambda_{j}\right|^{q} 2^{\left[\left(\beta+n \delta_{2}\right)-\alpha(0)\right] j \frac{q}{2}}+C \sup _{L>0, L \in \mathbb{Z}} 2^{-L \lambda q} \sum_{k=0}^{L} \sum_{j=0}^{k-1}\left|\lambda_{j}\right|^{q} 2^{(j-k)\left[\left(\beta+n \delta_{2}\right)-\alpha_{\infty}\right] \frac{q}{2}} \\
& \leq C\left[\sup _{L>0, L \in \mathbb{Z}} 2^{-L \lambda q} \sum_{j=-\infty}^{-1}\left|\lambda_{j}\right|^{q}+\sup _{L>0, L \in \mathbb{Z}} 2^{-L \lambda q} \sum_{k=0}^{L} \sum_{j=0}^{k-1}\left|\lambda_{j}\right|^{q} 2^{(j-k)\left[\left(\beta+n \delta_{2}\right)-\alpha_{\infty}\right] \frac{q}{2}}\right]
\end{aligned}
$$




$$
\begin{aligned}
& \leq C\left[\sup _{L>0, L \in \mathbb{Z}} 2^{-L \lambda q} \sum_{j=-\infty}^{-1}\left|\lambda_{j}\right|^{q}+\sup _{L>0, L \in \mathbb{Z}} 2^{-L \lambda q} \sum_{j=0}^{L-1}\left|\lambda_{j}\right|^{q} \sum_{k=j+1}^{L} 2^{(j-k)\left[\left(\beta+n \delta_{2}\right)-\alpha_{\infty}\right]^{q}}\right] \\
& \leq C\left[\sup _{L>0, L \in \mathbb{Z}} 2^{-L \lambda q} \sum_{j=-\infty}^{-1}\left|\lambda_{j}\right|^{q}+\sup _{L>0, L \in \mathbb{Z}} 2^{-L \lambda q} \sum_{j=0}^{L-1}\left|\lambda_{j}\right|^{q}\right] \\
& \leq C \sup _{L>0, L \in \mathbb{Z}} 2^{-L \lambda q} \sum_{j=-\infty}^{L-1}\left|\lambda_{j}\right|^{q} \leq C Y .
\end{aligned}
$$

The proof is completed.

Theorem 14. Suppose that $b \in \operatorname{BMO}\left(\mathbb{R}^{n}\right), p(\cdot) \in \mathcal{B}\left(\mathbb{R}^{n}\right)$ and let $\Omega \in L^{r}\left(\mathbb{S}^{n-1}\right)\left(r>p^{+}\right)$satisfies (7). Let $0<q<$ $\infty, 0 \leq \lambda<\infty$ and $\alpha(\cdot) \in L^{\infty}\left(\mathbb{R}^{n}\right)$ satisfies conditions (4) and (5) of Proposition 3. If $2 \lambda \leq \alpha(\cdot), n \delta_{2} \leq \alpha(0), \alpha_{\infty}<$ $\beta+n \delta_{2}$, then $\left[b, T_{\Omega}\right]$ is bounded from $H M \dot{K}_{p(\cdot), \lambda}^{\alpha(\cdot), q}$ or $\left(H M K_{p(\cdot), \lambda}^{\alpha(\cdot), q}\right)$ to $M \dot{K}_{p(\cdot), \lambda}^{\alpha(\cdot), q}$ or $\left(M K_{p(\cdot), \lambda}^{\alpha(\cdot), q}\right)$.

Proof. It suffices to prove for $\operatorname{HMK}_{p(\cdot), \lambda}^{\alpha(\cdot), q}$. Set $b \in \mathrm{BMO}\left(\mathbb{R}^{n}\right)$ and $h \in H M \dot{K}_{p(\cdot), \lambda}^{\alpha(\cdot), q}$. By Lemma 10, $h=$ $\sum_{j=-\infty}^{\infty} \lambda_{j} g_{j}$ converges in $\mathcal{S}^{\prime}\left(\mathbb{R}^{n}\right)$, where $\|h\|_{H M \dot{K}_{p(\cdot), \lambda}^{\alpha(\cdot), q}} \approx \inf _{L \in \mathbb{Z}} \sup ^{L \lambda}\left(\sum_{j=-\infty}^{L}\left|\lambda_{j}\right|^{q}\right)^{1 / q}$, and $g_{j}$ is a dyadic central $(\alpha(\cdot), p(\cdot))$-atom with support contained in $B_{j}$. For simplicity, we denote $Y=\sup _{L \in \mathbb{Z}} 2^{L \lambda} \sum_{j=-\infty}^{L}\left|\lambda_{j}\right|^{q}$. By virtue of Lemma 8, we rewrite

$$
\begin{aligned}
& \left\|T_{\Omega}^{b}(h)\right\|_{M \dot{K}_{p(\cdot), \lambda}^{\alpha(\cdot), q}\left(\mathbb{R}^{n}\right)} \approx \max \left\{\sup _{L \leq 0, L \in \mathbb{Z}} 2^{-L \lambda}\left(\sum_{k=-\infty}^{L} 2^{k \alpha(0) q}\left\|T_{\Omega}^{b}(h) \chi_{k}\right\|_{L^{p(\cdot)}\left(\mathbb{R}^{n}\right)}^{q}\right)^{1 / q},\right. \\
& \left.\sup _{L>0, L \in \mathbb{Z}}\left[2^{-L \lambda}\left(\sum_{k=-\infty}^{-1} 2^{k \alpha(0) q}\left\|T_{\Omega}^{b}(h) \chi_{k}\right\|_{L^{p(\cdot)}\left(\mathbb{R}^{n}\right)}^{q}\right)^{1 / q}+2^{-L \lambda}\left(\sum_{k=0}^{L} 2^{k \alpha_{\infty} q}\left\|T_{\Omega}^{b}(h) \chi_{k}\right\|_{L^{p(\cdot)}\left(\mathbb{R}^{n}\right)}^{q}\right)^{1 / q}\right]\right\} \\
& \approx \max \left\{E^{\prime}, F^{\prime}+G^{\prime}\right\}
\end{aligned}
$$

where

$$
\begin{aligned}
E^{\prime} & =\sup _{L \leq 0, L \in \mathbb{Z}} 2^{-L \lambda q} \sum_{k=-\infty}^{L} 2^{k \alpha(0) q}\left\|T_{\Omega}^{b}(h) \chi_{k}\right\|_{L^{p(\cdot)}\left(\mathbb{R}^{n}\right)}^{q}, \\
F^{\prime} & =\sum_{k=-\infty}^{-1} 2^{k \alpha(0) q}\left\|T_{\Omega}^{b}(h) \chi_{k}\right\|_{L^{p(\cdot)}\left(\mathbb{R}^{n}\right)^{\prime}}^{q} \\
G^{\prime} & =\sup _{L>0, L \in \mathbb{Z}} 2^{-L \lambda q} \sum_{k=0}^{L} 2^{k \alpha_{\infty} q}\left\|T_{\Omega}^{b}(h) \chi_{k}\right\|_{L^{p(\cdot)\left(\mathbb{R}^{n}\right)}}^{q} .
\end{aligned}
$$

To complete the prove, we only need to show that there exists a constant $C>0$, such that $E^{\prime}, F^{\prime}, G^{\prime} \leq C$. First we show that $E^{\prime} \leq C Y$.

$$
\begin{aligned}
E^{\prime} & =\sup _{L \leq 0, L \in \mathbb{Z}} 2^{-L \lambda q} \sum_{k=-\infty}^{L} 2^{k \alpha(0) q}\left\|T_{\Omega}^{b}(h) \chi_{k}\right\|_{L^{p(\cdot)}\left(\mathbb{R}^{n}\right)}^{q} \\
& \leq \sup _{L \leq 0, L \in \mathbb{Z}} 2^{-L \lambda q} \sum_{k=-\infty}^{L} 2^{k \alpha(0) q}\left(\sum_{j=k}^{\infty}\left|\lambda_{j}\right|\left\|T_{\Omega}^{b}\left(g_{j}\right) \chi_{k}\right\|_{L^{p(\cdot)}\left(\mathbb{R}^{n}\right)}\right)^{q} \\
& +\sup _{L \leq 0, L \in \mathbb{Z}} 2^{-L \lambda q} \sum_{k=-\infty}^{L} 2^{k \alpha(0) q}\left(\sum_{j=-\infty}^{k-1}\left|\lambda_{j}\right|\left\|T_{\Omega}^{b}\left(g_{j}\right) \chi_{k}\right\|_{L^{p(\cdot)}\left(\mathbb{R}^{n}\right)}\right)^{q} \\
& :=E_{1}^{\prime}+E_{2}^{\prime} .
\end{aligned}
$$


By the $\left(L^{p(\cdot)}\left(\mathbb{R}^{n}\right), L^{p(\cdot)}\left(\mathbb{R}^{n}\right)\right)$-boundedness of the $T_{\Omega}^{b}$ (see [13]) and following the same way as we estimated $E_{1}$ in Theorem 13, we get $E_{1}^{\prime} \leq C\|b\|_{*} Y$.

Now, we estimate $E_{2}^{\prime}$. For each $k \in \mathbb{Z}$ and $x \in A_{k}$, by Lemma 7 and Minkowski inequality, we get

$$
\begin{aligned}
\left\|T_{\Omega}^{b}\left(g_{j}\right) \chi_{k}\right\|_{L^{p(\cdot)}\left(\mathbb{R}^{n}\right)} \leq & \int_{B_{j}}\left\|\left|\frac{\Omega(\cdot-y)}{|\cdot-y|^{n}}-\frac{\Omega(\cdot)}{|\cdot|^{n}}\right|(b(\cdot)-b(y)) \chi_{k}\right\|_{L^{p(\cdot)}\left(\mathbb{R}^{n}\right)}\left|g_{j}(y)\right| \mathrm{d} y \\
\leq & \int_{B_{j}}\left\|\left|\frac{\Omega(\cdot-y)}{|\cdot-y|^{n}}-\frac{\Omega(\cdot)}{|\cdot|^{n}}\right|\left|b(\cdot)-b_{B_{j}}\right| \chi_{k}\right\|_{L^{p(\cdot)}\left(\mathbb{R}^{n}\right)}\left|g_{j}(y)\right| \mathrm{d} y \\
& +\int_{B_{j}}\left\|\left|\frac{\Omega(\cdot-y)}{|\cdot-y|^{n}}-\frac{\Omega(\cdot)}{|\cdot|^{n}}\right| \chi_{k}\right\|_{L^{p(\cdot)}\left(\mathbb{R}^{n}\right)}\left|b_{B_{j}}-b(y)\right|\left|g_{j}(y)\right| \mathrm{d} y .
\end{aligned}
$$

Since $\tilde{p}(\cdot)>1$ and $1 / p(\cdot)=1 / \tilde{p}(\cdot)+1 / r$. Since $r>p^{+}$, so by Lemma 4 and Lemma 11 , we get

$$
\begin{aligned}
\left\|T_{\Omega}^{b}\left(g_{j}\right) \chi_{k}\right\|_{L^{p(\cdot)}\left(\mathbb{R}^{n}\right)} \leq & \left\|\frac{\Omega(\cdot-y)}{|\cdot-y|^{n}}-\frac{\Omega(\cdot)}{|\cdot|^{n}}\left|\left\|_{L^{r}\left(\mathbb{R}^{n}\right)}\right\| b(\cdot)-b_{B_{j}} \chi_{k} \|_{L^{\tilde{p}(\cdot)}\left(\mathbb{R}^{n}\right)} \int_{B_{j}}\right| g_{j}(y) \mid d y\right. \\
& +\left\|\frac{\Omega(\cdot-y)}{|\cdot-y|^{n}}-\frac{\Omega(\cdot)}{|\cdot|^{n}}\left|\left\|_{L^{r}\left(\mathbb{R}^{n}\right)}\right\| \chi_{B_{k}} \|_{L^{\tilde{p}(\cdot)}\left(\mathbb{R}^{n}\right)} \int_{B_{j}}\right| b_{B_{j}}-b(y)|| g_{j}(y) \mid d y\right.
\end{aligned}
$$

From (8) and Lemmas 4-6, we get

$$
\begin{aligned}
& \left\|T_{\Omega}^{b}\left(g_{j}\right) \chi_{k}\right\|_{L^{p(\cdot)}\left(\mathbb{R}^{n}\right)} \leq C\left\|\left|\frac{\Omega(\cdot-y)}{|\cdot-y|^{n}}-\frac{\Omega(\cdot)}{|\cdot|^{n}}\right|\right\|_{L^{r}\left(\mathbb{R}^{n}\right)}(k-j)\|b\|_{*}\left(\left\|\chi_{B_{k}}\right\|_{L^{p(\cdot)}\left(\mathbb{R}^{n}\right)}\left|B_{k}\right|^{\frac{1}{r}}\right)\left\|g_{j}\right\|_{L^{p(\cdot)}\left(\mathbb{R}^{n}\right)} \\
& +\left\|\left|\frac{\Omega(\cdot-y)}{|\cdot-y|^{n}}-\frac{\Omega(\cdot)}{|\cdot|^{n}}\right|\right\|_{L^{r}\left(\mathbb{R}^{n}\right)}\left\|\left(b_{B_{j}}-b\right) \chi_{B_{j}}\right\|_{L^{\left.p^{(} \cdot()\right)\left(\mathbb{R}^{n}\right)}}\left(\left\|\chi_{B_{k}}\right\|_{L^{p(\cdot)}\left(\mathbb{R}^{n}\right)}\left|B_{k}\right|^{\frac{1}{r}}\right)\left\|g_{j}\right\|_{L^{p(\cdot)}\left(\mathbb{R}^{n}\right)} \\
& \leq C\left\|\left|\frac{\Omega(\cdot-y)}{|\cdot-y|^{n}}-\frac{\Omega(\cdot)}{|\cdot|^{n}}\right|\right\|_{L^{r}\left(\mathbb{R}^{n}\right)}(k-j)\|b\|_{*}\left(\left\|\chi_{B_{k}}\right\|_{L^{p(\cdot)}\left(\mathbb{R}^{n}\right)}\left|B_{k}\right|^{\frac{1}{r}}\right)\left\|g_{j}\right\|_{L^{p(\cdot)}\left(\mathbb{R}^{n}\right)} \\
& +\left\|\left|\frac{\Omega(\cdot-y)}{|\cdot-y|^{n}}-\frac{\Omega(\cdot)}{|\cdot|^{n} \mid}\right|\right\|_{L^{r}\left(\mathbb{R}^{n}\right)}\|b\|_{*}\left(\left\|\chi_{B_{k}}\right\|_{L^{p(\cdot)}\left(\mathbb{R}^{n}\right)}\left|B_{k}\right|^{\frac{1}{r}}\right)\left\|g_{j}\right\|_{L^{p(\cdot)}\left(\mathbb{R}^{n}\right)}\left\|\chi_{B_{j}}\right\|_{L^{p^{\prime}(\cdot)\left(\mathbb{R}^{n}\right)}} \\
& \leq C\|b\|_{*}(k-j) 2^{-n k+(j-k) \beta}\left(\left\|\chi_{B_{k}}\right\|_{L^{p^{\prime}(\cdot)}\left(\mathbb{R}^{n}\right)}^{-1}\left|B_{k}\right|\right)\left\|g_{j}\right\|_{L^{p(\cdot)}\left(\mathbb{R}^{n}\right)}\left\|\chi_{B_{j}}\right\|_{L^{p^{\prime}(\cdot)}\left(\mathbb{R}^{n}\right)} \\
& \leq C\|b\|_{*}(k-j) 2^{(j-k) \beta}\left(\frac{\left\|\chi_{B_{j}}\right\|_{L^{p^{\prime}(\cdot)\left(\mathbb{R}^{n}\right)}}}{\left\|\chi_{B_{k}}\right\|_{L^{p^{\prime}(\cdot)}\left(\mathbb{R}^{n}\right)}}\right)\left\|g_{j}\right\|_{L^{p(\cdot)}\left(\mathbb{R}^{n}\right)} \\
& \leq C\|b\|_{*}(k-j) 2^{-j \alpha_{j}+(j-k)\left(\beta+n \delta_{2}\right)} \text {. }
\end{aligned}
$$

Therefore, when $0<q \leq 1$, we obtain

$$
\begin{aligned}
E_{2}^{\prime} & =\sup _{L \leq 0, L \in \mathbb{Z}} 2^{-L \lambda q} \sum_{k=-\infty}^{L} 2^{k \alpha(0) q}\left(\sum_{j=-\infty}^{k-1}\left|\lambda_{j}\right|\left\|T_{\Omega}^{b}\left(g_{j}\right) \chi_{k}\right\|_{L^{p(\cdot)}\left(\mathbb{R}^{n}\right)}\right)^{q} \\
& \leq C\|b\|_{*}^{q} \sup _{L \leq 0, L \in \mathbb{Z}} 2^{-L \lambda q} \sum_{k=-\infty}^{L} 2^{k \alpha(0) q}\left(\sum_{j=-\infty}^{k-1}\left|\lambda_{j}\right|(k-j) 2^{(j-k)\left(\beta+n \delta_{2}\right)-j \alpha(0)}\right)^{q} \\
& \leq C\|b\|_{*}^{q} \sup _{L \leq 0, L \in \mathbb{Z}} 2^{-L \lambda q} \sum_{k=-\infty}^{L}\left(\sum_{j=-\infty}^{k-1}\left|\lambda_{j}\right|(k-j) 2^{\left[(j-k)\left(\beta+n \delta_{2}\right)-\alpha(0)\right]}\right)^{q} \\
& \leq C\|b\|_{*}^{q} \sup _{L \leq 0, L \in \mathbb{Z}} 2^{-L \lambda q} \sum_{j=-\infty}^{L}\left|\lambda_{j}\right|^{q}\left(\sum_{k=j+1}^{-1}(k-j) 2^{\left[(j-k)\left(\beta+n \delta_{2}\right)-\alpha(0)\right]}\right)^{q} \\
& \leq C\|b\|_{*}^{q} \mathrm{Y} .
\end{aligned}
$$


When $0<q \leq \infty$, and $1 / q+1 / q^{\prime}=1$, by $n \delta_{2} \leq \alpha(0)<\beta+n \delta_{2}$ and Hölder's inequality, we have

$$
\begin{aligned}
E_{2}^{\prime} & =\sup _{L \leq 0, L \in \mathbb{Z}} 2^{-L \lambda q} \sum_{k=-\infty}^{L} 2^{k \alpha(0) q}\left(\sum_{j=-\infty}^{k-1}\left|\lambda_{j}\right|\left\|T_{\Omega}^{b}\left(g_{j}\right) \chi_{k}\right\|_{L^{p(\cdot)}\left(\mathbb{R}^{n}\right)}\right)^{q} \\
& \leq C\|b\|_{*}^{q} \sup _{L \leq 0, L \in \mathbb{Z}} 2^{-L \lambda q} \sum_{k=-\infty}^{L}\left(\sum_{j=-\infty}^{k-1}\left|\lambda_{j}\right|^{q} 2^{\left[(j-k)\left(\beta+n \delta_{2}\right)-\alpha(0)\right]}\right]^{q} \\
& \times\left(\sum_{j=-\infty}^{k-1}(k-j)^{q^{\prime}} 2^{\left[(j-k)\left(\beta+n \delta_{2}\right)-\alpha(0)\right]^{\frac{q^{\prime}}{2}}}\right)^{\frac{q}{q^{\prime}}} \\
& \leq C\|b\|_{*}^{q} \sup _{L \leq 0, L \in \mathbb{Z}} 2^{-L \lambda q} \sum_{j=-\infty}^{L}\left|\lambda_{j}\right|^{q} \sum_{k=j+1}^{-1} 2^{\left[(j-k)\left(\beta+n \delta_{2}\right)-\alpha(0)\right] \frac{q}{2}} \\
& \leq C\|b\|_{*}^{q} \mathrm{Y} .
\end{aligned}
$$

Now we prove that $F^{\prime} \leq C Y$.

$$
\begin{aligned}
F^{\prime}= & \sum_{k=-\infty}^{-1} 2^{k \alpha(0) q}\left\|T_{\Omega}^{b}(h) \chi_{k}\right\|_{L^{p(\cdot)}\left(\mathbb{R}^{n}\right)}^{q} \leq \sum_{k=-\infty}^{-1} 2^{k \alpha(0) q}\left(\sum_{j=k}^{\infty}\left|\lambda_{j}\right|\left\|T_{\Omega}^{b}\left(g_{j}\right) \chi_{k}\right\|_{L^{p(\cdot)}\left(\mathbb{R}^{n}\right)}\right)^{q} \\
& +\sum_{k=-\infty}^{-1} 2^{k \alpha(0) q}\left(\sum_{j=-\infty}^{k-1}\left|\lambda_{j}\right|\left\|T_{\Omega}^{b}\left(g_{j}\right) \chi_{k}\right\|_{L^{p(\cdot)}\left(\mathbb{R}^{n}\right)}\right)^{q} \\
:= & F_{1}^{\prime}+F_{2}^{\prime} .
\end{aligned}
$$

To estimate $F_{1}^{\prime}$. By the boundedness of the $T_{\Omega}^{b}$ on $L^{p(\cdot)}\left(\mathbb{R}^{n}\right)$ (see[13]) and following the same way as we estimated $F_{1}$ in Theorem 13, we get

$$
F_{1}^{\prime} \leq C\|b\|_{*}^{q} \mathrm{Y} .
$$

For $F_{2}$, when $0<q \leq 1$, by inequality (18) and $n \delta_{2} \leq \alpha(0)<\varepsilon+n \delta_{2}$, we have

$$
\begin{aligned}
F_{2}^{\prime}= & \sum_{k=-\infty}^{-1} 2^{k \alpha(0) q}\left(\sum_{j=-\infty}^{k-1}\left|\lambda_{j}\right|\left\|T_{\Omega}^{b}\left(g_{j}\right) \chi_{k}\right\|_{L^{p(\cdot)}\left(\mathbb{R}^{n}\right)}\right)^{q} \\
& \leq C\|b\|_{*}^{q} \sum_{k=-\infty}^{-1} 2^{k \alpha(0) q}\left(\sum_{j=-\infty}^{k-1}\left|\lambda_{j}\right|(k-j) 2^{(j-k)\left(\beta+n \delta_{2}\right)-j \alpha(0)}\right)^{q} \\
& \leq C\|b\|_{*}^{q} \sum_{k=-\infty}^{-1}\left(\sum_{j=-\infty}^{k-1}\left|\lambda_{j}\right|(k-j) 2^{\left[(j-k)\left(\beta+n \delta_{2}\right)-\alpha(0)\right]}\right)^{q} \\
& \leq C\|b\|_{*}^{q} \sum_{j=-\infty}^{-1}\left|\lambda_{j}\right|^{q}\left(\sum_{k=j+1}^{-1}(k-j) 2^{\left[(j-k)\left(\beta+n \delta_{2}\right)-\alpha(0)\right]}\right)^{q} \\
& \leq C\|b\|_{*}^{q} \mathrm{Y} .
\end{aligned}
$$

when $0<q \leq \infty$, and $1 / q+1 / q^{\prime}=1$. by $n \delta_{2} \leq \alpha(0)<\beta+n \delta_{2}$ and Hölder's inequality, we obtain

$$
\begin{aligned}
F_{2}^{\prime} & =\sum_{k=-\infty}^{-1} 2^{k \alpha(0) q}\left(\sum_{j=-\infty}^{k-1}\left|\lambda_{j}\right|\left\|T_{\Omega}^{b}\left(g_{j}\right) \chi_{k}\right\|_{L^{p(\cdot)}\left(\mathbb{R}^{n}\right)}\right)^{q} \\
& \left.\leq C\|b\|_{*}^{q} \sum_{k=-\infty}^{-1}\left(\sum_{j=-\infty}^{k-1}\left|\lambda_{j}\right|^{q} 2^{\left[(j-k)\left(\beta+n \delta_{2}\right)-\alpha(0)\right]}\right)^{q}\right) \times\left(\sum_{j=-\infty}^{k-1}(k-j)^{q^{\prime}} 2^{\left[(j-k)\left(\beta+n \delta_{2}\right)-\alpha(0)\right] \frac{q^{\prime}}{2}}\right)^{\frac{q}{q^{\prime}}} \\
& \leq C\|b\|_{*}^{q} \sum_{j=-\infty}^{-1}\left|\lambda_{j}\right|^{q} \sum_{k=j+1}^{-1} 2^{\left[(j-k)\left(\beta+n \delta_{2}\right)-\alpha(0)\right] \frac{q}{2}} \\
& \leq C\|b\|_{*}^{q} \mathrm{Y} .
\end{aligned}
$$


Finally, we show that $G^{\prime} \leq C Y$.

$$
\begin{aligned}
G^{\prime}= & \sup _{L>0, L \in \mathbb{Z}} 2^{-L \lambda q} \sum_{k=0}^{L} 2^{k \alpha_{\infty} q}\left\|T_{\Omega}^{b}(h) \chi_{k}\right\|_{L^{p(\cdot)}\left(\mathbb{R}^{n}\right)^{\prime}}^{q} \\
\leq & \sup _{L>0, L \in \mathbb{Z}} 2^{-L \lambda q} \sum_{k=0}^{L} 2^{k \alpha_{\infty} q}\left(\sum_{j=k}^{\infty}\left|\lambda_{j}\right|\left\|T_{\Omega}^{b}\left(g_{j}\right) \chi_{k}\right\|_{L^{p(\cdot)}\left(\mathbb{R}^{n}\right)}\right)^{q} \\
& \quad+\sup _{L>0, L \in \mathbb{Z}} 2^{-L \lambda q} \sum_{k=0}^{L} 2^{k \alpha_{\infty} q}\left(\sum_{j=-\infty}^{k-1}\left|\lambda_{j}\right|\left\|T_{\Omega}^{b}\left(g_{j}\right) \chi_{k}\right\|_{L^{p(\cdot)}\left(\mathbb{R}^{n}\right)}\right)^{q}, \\
:= & G_{1}^{\prime}+G_{2}^{\prime} .
\end{aligned}
$$

For $G_{1}^{\prime}$, by the boundedness of the commutator $T_{\Omega}^{b}$ in $L^{p(\cdot)}\left(\mathbb{R}^{n}\right)$ (see [13]), and following the same way as we estimated $G_{1}$ in Theorem 13, we get

$$
G_{1}^{\prime} \leq C\|b\|_{*}^{q} \mathrm{Y} .
$$

Now, we estimate $G_{2}^{\prime}$. When $0<q<\infty$, and $1 / q+1 / q^{\prime}=1$, from inequality (18), since $n \delta_{2} \leq \alpha(0), \alpha_{\infty}<$ $\beta+n \delta_{2}$ and applying Hölder's inequality, we obtain

$$
\begin{aligned}
& G_{2}^{\prime}=\sup _{L>0, L \in \mathbb{Z}} 2^{-L \lambda q} \sum_{k=0}^{L} 2^{k \alpha_{\infty} q}\left(\sum_{j=-\infty}^{k-1}\left|\lambda_{j}\right|\left\|T_{\Omega}^{b}\left(g_{j}\right) \chi_{k}\right\|_{L^{p(\cdot)}\left(\mathbb{R}^{n}\right)}\right)^{q} \\
& \leq C\|b\|_{*} \sup _{L>0, L \in \mathbb{Z}} 2^{-L \lambda q} \sum_{k=0}^{L} 2^{k \alpha_{\infty} q}\left(\sum_{j=-\infty}^{k-1}\left|\lambda_{j}\right|(k-j) 2^{(j-k)\left(\beta+n \delta_{2}\right)-j \alpha_{j}}\right)^{q} \\
& \leq C\|b\|_{*} \sup _{L>0, L \in \mathbb{Z}} 2^{-L \lambda q} \sum_{k=0}^{L} 2^{k \alpha_{\infty} q}\left(\sum_{j=-\infty}^{-1}\left|\lambda_{j}\right|(k-j) 2^{(j-k)\left(\beta+n \delta_{2}\right)-j \alpha(0)}\right)^{q} \\
& +C\|b\|_{*} \sup _{L>0, L \in \mathbb{Z}} 2^{-L \lambda q} \sum_{k=0}^{L} 2^{k \alpha_{\infty} q}\left(\sum_{j=0}^{k-1}\left|\lambda_{j}\right|(k-j) 2^{(j-k)\left(\beta+n \delta_{2}\right)-j \alpha_{\infty}}\right)^{q} \\
& \leq C\|b\|_{*} \sup _{L>0, L \in \mathbb{Z}} 2^{-L \lambda q} \sum_{k=0}^{L} 2^{\left[\alpha_{\infty}-\left(\beta+n \delta_{2}\right)\right] k q}\left(\sum_{j=-\infty}^{-1}\left|\lambda_{j}\right|(k-j) 2^{\left[\left(\beta+n \delta_{2}\right)-\alpha(0)\right] j}\right)^{q} \\
& +C\|b\|_{*} \sup _{L>0, L \in \mathbb{Z}} 2^{-L \lambda q} \sum_{k=0}^{L}\left(\sum_{j=0}^{k-1}\left|\lambda_{j}\right|(k-j) 2^{(j-k)\left[\left(\beta+n \delta_{2}\right)-\alpha_{\infty}\right]}\right)^{q} \\
& \leq C\|b\|_{*} \sup _{L>0, L \in \mathbb{Z}} 2^{-L \lambda q} \sum_{k=0}^{L} 2^{\left[\alpha_{\infty}-\left(\beta+n \delta_{2}\right)\right] k q}\left(\sum_{j=-\infty}^{-1}\left|\lambda_{j}\right| 2^{j \frac{\left[\left(\beta+n \delta_{2}\right)-\alpha(0)\right]}{2}}\right)^{q}\left(\sum_{j=-\infty}^{-1}(k-j)^{q^{\prime}} 2^{\left.j\left[\left(\beta+n \delta_{2}\right)-\alpha(0)\right]\right]^{q^{\prime}}}\right)^{\frac{q}{q^{\prime}}} \\
& +C\|b\|_{*} \sup _{L>0, L \in \mathbb{Z}} 2^{-L \lambda q} \sum_{k=0}^{L}\left(\sum_{j=0}^{k-1}\left|\lambda_{j}\right| 2^{(j-k) \frac{\left[\left(\beta+n \delta_{2}\right)-\alpha_{\infty}\right]}{2}}\right)^{q}\left(\sum_{j=0}^{k-1}(k-j)^{q^{\prime}} 2^{(j-k)\left[\left(\beta+n \delta_{2}\right)-\alpha_{\infty}\right] \frac{q^{\prime}}{2}}\right)^{\frac{q}{q^{\prime}}} \\
& \leq C\|b\|_{*} \sup _{L>0, L \in \mathbb{Z}} 2^{-L \lambda q}\left(\sum_{j=-\infty}^{-1}\left|\lambda_{j}\right|^{q} 2^{\left[\left(\beta+n \delta_{2}\right)-\alpha(0)\right] j \frac{q}{2}}\right)+C\|b\|_{*} \sup _{L>0, L \in \mathbb{Z}} 2^{-L \lambda q} \sum_{k=0}^{L} \sum_{j=0}^{k-1}\left|\lambda_{j}\right|^{q} 2^{(j-k)\left[\left(\beta+n \delta_{2}\right)-\alpha_{\infty}\right] \frac{q}{2}} \\
& \leq C\|b\|_{*}\left[\sup _{L>0, L \in \mathbb{Z}} 2^{-L \lambda q} \sum_{j=-\infty}^{-1}\left|\lambda_{j}\right|^{q}+\sup _{L>0, L \in \mathbb{Z}} 2^{-L \lambda q} \sum_{k=0}^{L} \sum_{j=0}^{k-1}\left|\lambda_{j}\right|^{q} 2^{(j-k)\left[\left(\beta+n \delta_{2}\right)-\alpha_{\infty}\right] \frac{q}{2}}\right] \\
& \leq C\|b\|_{*}\left[\sup _{L>0, L \in \mathbb{Z}} 2^{-L \lambda q} \sum_{j=-\infty}^{-1}\left|\lambda_{j}\right|^{q}+\sup _{L>0, L \in \mathbb{Z}} 2^{-L \lambda q} \sum_{j=0}^{L-1}\left|\lambda_{j}\right|^{q} \sum_{k=j+1}^{L} 2^{(j-k)\left[\left(\beta+n \delta_{2}\right)-\alpha_{\infty}\right] \frac{q}{2}}\right] \\
& \leq C\|b\|_{*}\left[\sup _{L>0, L \in \mathbb{Z}} 2^{-L \lambda q} \sum_{j=-\infty}^{-1}\left|\lambda_{j}\right|^{q}+\sup _{L>0, L \in \mathbb{Z}} 2^{-L \lambda q} \sum_{j=0}^{L-1}\left|\lambda_{j}\right|^{q}\right] \\
& \leq C\|b\|_{*} \sup _{L>0, L \in \mathbb{Z}} 2^{-L \lambda q} \sum_{j=-\infty}^{L-1}\left|\lambda_{j}\right|^{q} \\
& \leq C\|b\|_{*} \mathrm{Y} \text {. }
\end{aligned}
$$


The proof is completed.

Theorem 15. Suppose that $b \in \dot{\Lambda}_{\gamma}\left(\mathbb{R}^{n}\right)(0<\gamma \leq 1), p_{1}(\cdot), p_{2}(\cdot) \in \mathcal{B}\left(\mathbb{R}^{n}\right)$ be such that $p_{1}^{+}<n / \gamma, 1 / p_{1}(x)-$ $1 / p_{2}(x)=\gamma / n, \Omega \in L^{r}\left(\mathbb{S}^{n-1}\right)\left(r>q_{2}^{+}\right)$with $1 \leq r^{\prime}<p_{1}^{-}$and satisfies

$$
\int_{0}^{1} \frac{w_{r}(\delta)}{\delta^{1+\gamma}} d \delta<\infty
$$

Let $0<q<\infty, 0 \leq \lambda<\infty$ and $\alpha(\cdot) \in L^{\infty}\left(\mathbb{R}^{n}\right)$ satisfies conditions (4) and (5) of Proposition 3. If $2 \lambda \leq \alpha(\cdot), n \delta_{2} \leq$ $\alpha(0), \alpha_{\infty}<\gamma+n \delta_{2}$, then $\left[b, T_{\Omega}\right]$ is bounded from $\operatorname{HMK}_{p_{1}(\cdot), \lambda}^{\alpha(\cdot), q}$ or $\left(\operatorname{HMK}_{p_{1}(\cdot), \lambda}^{\alpha(\cdot), q}\right)$ to $M \dot{K}_{p_{2}(\cdot), \lambda}^{\alpha(\cdot), q}$ or $\left(M K_{p_{2}(\cdot), \lambda}^{\alpha(\cdot), q}\right)$.

Proof. The prove of this Theorem follows almost similarly to that of Theorem 14. Instead of giving all details, we only give the modifications required for the estimation of $E^{\prime \prime}, F^{\prime \prime}$ and $G^{\prime \prime}$.

Note that if $x \in B_{k}$ for each $k \in \mathbb{Z}, y \in B_{j}$ and $j \leq k-1$. Let $\tilde{p}(\cdot)>1$ and $1 / p(\cdot)=1 / \tilde{p}(\cdot)+1 / r$, since $r>p^{+}$, so by Lemmas 10 and 12 , we get

$$
\begin{aligned}
\left\|T_{\Omega}^{b}\left(g_{j}\right) \chi_{k}\right\|_{L^{p(\cdot)\left(\mathbb{R}^{n}\right)} \leq} & \int_{B_{j}}\left\|\frac{\Omega(\cdot-y)}{|\cdot-y|^{n}}-\frac{\Omega(\cdot)}{|\cdot|^{n}}\left|(b(\cdot)-b(y)) \chi_{k} \|_{L^{p_{2}(\cdot)}\left(\mathbb{R}^{n}\right)}\right| g_{j}(y) \mid d y\right. \\
\leq & \int_{B_{j}}\left\|\frac{\Omega(\cdot-y)}{|\cdot-y|^{n}}-\frac{\Omega(\cdot)}{|\cdot|^{n}}\left|b(\cdot)-b_{B_{j}}\right| \chi_{k}\right\|_{L^{p_{2}(\cdot)}\left(\mathbb{R}^{n}\right)}\left|g_{j}(y)\right| d y \\
& +\int_{B_{j}}\left\|\frac{\Omega(\cdot-y)}{|\cdot-y|^{n}}-\frac{\Omega(\cdot)}{|\cdot|^{n}}\left|\chi_{k} \|_{L^{p_{2}(\cdot)}\left(\mathbb{R}^{n}\right)}\right| b_{B_{j}}-b(y)|| g_{j}(y) \mid d y\right.
\end{aligned}
$$

Since $\tilde{p_{2}}(\cdot)>1$ and $1 / p_{2}(\cdot)=1 / \tilde{p_{2}}(\cdot)+1 / r$, by $r>p^{+}$and Lemmas 11 and 12 , we deduced

$$
\begin{aligned}
\left\|T_{\Omega}^{b}\left(g_{j}\right) \chi_{k}\right\|_{L^{p_{2}(\cdot)}\left(\mathbb{R}^{n}\right)} \leq & \left\|\frac{\Omega(\cdot-y)}{|\cdot-y|^{n}}-\frac{\Omega(\cdot)}{|\cdot|^{n}}\left|\left\|_{L^{r}\left(\mathbb{R}^{n}\right)}\right\|\left(b-b_{B_{j}}\right) \chi_{k} \|_{L^{\tilde{p}_{2}(\cdot)}\left(\mathbb{R}^{n}\right)} \int_{B_{j}}\right| g_{j}(y) \mid d y\right. \\
& +\left\|\frac{\Omega(\cdot-y)}{|\cdot-y|^{n}}-\frac{\Omega(\cdot)}{|\cdot|^{n}}\left|\| _ { L ^ { r } ( \mathbb { R } ^ { n } ) } \| \chi _ { B _ { k } } \left\|_{L^{\tilde{p}_{2}(\cdot)}\left(\mathbb{R}^{n}\right)} \int_{B_{j}}\left|b_{B_{j}}-b(y) \| g_{j}(y)\right| d y\right.\right.\right. \\
\leq & C\left\|\left|\frac{\Omega(\cdot-y)}{|\cdot-y|^{n}}-\frac{\Omega(\cdot)}{|\cdot|^{n}}\right|\right\|_{L^{r}\left(\mathbb{R}^{n}\right)}\left\|\left(b-b_{B_{j}}\right) \chi_{k}\right\|_{L^{\tilde{p}_{2}(\cdot)}\left(\mathbb{R}^{n}\right)}\left\|g_{j}\right\|_{L^{p_{1}(\cdot)}\left(\mathbb{R}^{n}\right)}\left\|\chi_{j}\right\|_{L^{p_{1}^{\prime}(\cdot)}\left(\mathbb{R}^{n}\right)} \\
& +\left\|\frac{\Omega(\cdot-y)}{|\cdot-y|^{n}}-\frac{\Omega(\cdot)}{|\cdot|^{n}} \mid\right\|_{L^{r}\left(\mathbb{R}^{n}\right)}\left\|\chi_{B_{k}}\right\|_{L^{\tilde{p}_{2}(\cdot)}\left(\mathbb{R}^{n}\right)}\left\|\left(b-b_{B_{j}}\right) \chi_{B_{j}}\right\|_{L^{p_{1}^{\prime}(\cdot)}\left(\mathbb{R}^{n}\right)}\left\|g_{j}\right\|_{L^{p_{1}(\cdot)}\left(\mathbb{R}^{n}\right)^{n}} .
\end{aligned}
$$

By Lemma 9, we have

$$
\begin{aligned}
\left\|\frac{\Omega(\cdot-y)}{|\cdot-y|^{n}}-\frac{\Omega(\cdot)}{|\cdot|^{n}}\right\|_{L^{r}\left(\mathbb{R}^{n}\right)} & \leq C 2^{(k-1)\left(\frac{n}{r}-n\right)}\left\{\frac{|y|}{2^{k-1}}+\int_{|y| / 2^{k}}^{|y| / 2^{k-1}} \frac{w_{r}(\delta)}{\delta} d \delta\right\} \\
& \leq C 2^{(k-1)\left(\frac{n}{r}-n\right)}\left(2^{j-k}+2^{(j-k) \gamma} \int_{0}^{1} \frac{w_{r}(\delta)}{\delta^{1+\gamma}} d \delta\right) \\
& \leq C 2^{(k-1)\left(\frac{n}{r}-n\right)} 2^{(j-k) \gamma} .
\end{aligned}
$$

by Lemma 4-6, we have

$$
\begin{aligned}
& \left\|T_{\Omega}^{b}\left(g_{j}\right) \chi_{k}\right\|_{L^{p_{2}(\cdot)}\left(\mathbb{R}^{n}\right)} \leq C 2^{(k-1)\left(\frac{n}{s}-n\right)+(j-k) \gamma}\|b\|_{\dot{\Lambda}_{\gamma}\left(\mathbb{R}^{n}\right)} 2^{\gamma k}\left(\frac{\left\|\chi_{B_{k}}\right\|_{L^{p_{1}(\cdot)}\left(\mathbb{R}^{n}\right)}}{\left|B_{k}\right|^{\frac{1}{r}+\frac{\gamma}{n}}}\right)\left\|\chi_{j}\right\|_{L^{p_{1}^{\prime}(\cdot)}\left(\mathbb{R}^{n}\right)}\left\|g_{j}\right\|_{L^{p_{1}(\cdot)}\left(\mathbb{R}^{n}\right)} \\
& +C 2^{(k-1)\left(\frac{n}{s}-n\right)+(j-k) \gamma}\|b\|_{\Lambda_{\gamma}\left(\mathbb{R}^{n}\right)} 2^{\gamma k}\left(\frac{\left\|\chi_{B_{k}}\right\|_{L^{p_{1}(\cdot)}\left(\mathbb{R}^{n}\right)}}{\left|B_{k}\right|^{\frac{1}{r}+\frac{\gamma}{n}}}\right)\left\|\chi_{j}\right\|_{L^{p_{1}^{\prime}(\cdot)}\left(\mathbb{R}^{n}\right)}\left\|g_{j}\right\|_{L^{p_{1}(\cdot)}\left(\mathbb{R}^{n}\right)} \\
& \leq C\|b\|_{\Lambda_{\gamma}\left(\mathbb{R}^{n}\right)} 2^{-n k+(j-k) \gamma}\left(\left\|\chi_{B_{k}}\right\|_{L^{p_{1}^{(}(\cdot)}\left(\mathbb{R}^{n}\right)}^{-}\left|B_{k}\right|\right)\left\|\chi_{j}\right\|_{L^{p_{1}^{\prime}(\cdot)}\left(\mathbb{R}^{n}\right)}\left\|g_{j}\right\|_{L^{p_{1}(\cdot)}\left(\mathbb{R}^{n}\right)}
\end{aligned}
$$




$$
\begin{aligned}
& \leq C\|b\|_{\dot{\Lambda}_{\gamma}\left(\mathbb{R}^{n}\right)} 2^{(j-k) \gamma}\left(\frac{\left\|\chi_{j}\right\|_{L^{p_{1}^{\prime}(\cdot)}\left(\mathbb{R}^{n}\right)}}{\left\|\chi_{B_{k}}\right\|_{L^{p_{1}^{\prime}(\cdot)}\left(\mathbb{R}^{n}\right)}}\right)\left\|g_{j}\right\|_{L^{p_{1}(\cdot)\left(\mathbb{R}^{n}\right)}} \\
& \leq C\|b\|_{\dot{\Lambda}_{\gamma}\left(\mathbb{R}^{n}\right)} 2^{(j-k)\left(\gamma+n \delta_{2}\right)-j \alpha_{j}} .
\end{aligned}
$$

From this, following the same calculations as we did for $E^{\prime}, F^{\prime}$ and $G^{\prime}$ in Theorem 14 , we get

$$
E^{\prime \prime}, F^{\prime \prime}, G^{\prime \prime} \leq C\|b\|_{\dot{\Lambda}_{\gamma}\left(\mathbb{R}^{n}\right)} \mathrm{Y} .
$$

Acknowledgments: This paper is supported by Shendi University.

Author Contributions: All authors contributed equally to the writing of this paper. All authors read and approved the final manuscript.

Conflicts of Interest: "The authors declare no conflict of interest."

\section{References}

[1] Calderón, A. P., \& Zygmund, A. (1952). On the existence of certain singular integrals. Acta Mathematica, 88(1), 85-139.

[2] Calderón, A. P., \& Zygmund, A. (1956). On singular integrals. American Journal of Mathematics, 78(2), 289-309.

[3] Coifman, R. R., Rochberg, R., \& Weiss, G. (1976). Factorization theorems for Hardy spaces in several variables. Annals of Mathematics, 611-635.

[4] Shanzhen, L., Yong, D., \& Dunyan, Y. (2007). Singular integrals and related topics. World Scientific.

[5] Kováčik, O., \& Rákosník, J. (1991). On spaces $L^{p(\cdot)}$ and $W^{k, p(\cdot)}$, Czechoslovak Math., 41, 592-618.

[6] Almeida, A., \& Hästö, P. (2010). Besov spaces with variable smoothness and integrability. Journal of Functional Analysis, 258(5), 1628-1655.

[7] Almeida, A., Hasanov, J., \& Samko, S. (2008). Maximal and potential operators in variable exponent Morrey spaces. Georgian Mathematical Journal, 15(2), 195-208.

[8] Izuki, M. (2010). Boundedness of commutators on Herz spaces with variable exponent. Rendiconti del Circolo Matematico di Palermo, 59(2), 199-213.

[9] Izuki, M. (2010). Fractional integrals on Herz-Morrey spaces with variable exponent. Hiroshima Mathematical Journal, 40(3), 343-355.

[10] Lijuan, W. A. N. G., \& Tao, S. (2016). Parameterized Littlewood-Paley operators and their commutators on Herz spaces with variable exponents. Turkish Journal of Mathematics, 40(1), 122-145.

[11] Xu, J., \& Yang, X. (2015). Herz-Morrey-Hardy spaces with variable exponents and their applications. Journal of Function Spaces, 2015, ID.160635.

[12] Cruz-Uribe, D., Fiorenza, A., Martell, J. M., \& Pérez, C. (2006). The boundedness of classical operators on variable $L^{p}$ spaces, Ann. Acad. Sci. Fenn. Math., 31, 239-264.

[13] Wang, H. (2017). Commutators of singular integuler operator on Herz-Type Hardy spaces with variable exponents, J. Korean Math. Soc, 54, 713-732.

[14] Capone, C., David Cruz-Uribe, S. F. O., \& Fiorenza, A. (2007). The fractional maximal operator and fractional integrals on variable $L^{p}$ spaces. Revista Matemática Iberoamericana, 23(3), 743-770.

[15] Diening, L., \& Ruzicka, M. (2003). Calderón-Zygmund operators on generalized Lebesgue spaces $L^{p(\cdot)}$ and problems related to fluid dynamics, J. Reine. Angew. Math., 563, 197-220.

[16] Diening, L. (2002). Maximal function on generalized Lebesgue spaces $L^{p(x)}$. Math Inequal Appl, 7, 245-253.

[17] Izuki, M. (2010). Boundedness of sublinear operators on Herz spaces with variable exponent and application to wavelet characterization. Analysis Mathematica, 36(1), 33-50.

[18] Almeida, A., \& Drihem, D. (2012). Maximal, potential and singular type operators on Herz spaces with variable exponents. Journal of Mathematical Analysis and Applications, 394(2), 781-795.

[19] Cruz-Uribe, D. V., \& Fiorenza, A. (2013). Variable Lebesgue spaces: Foundations and harmonic analysis. Springer Science \& Business Media.

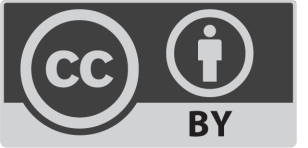

(C) 2019 by the authors; licensee PSRP, Lahore, Pakistan. This article is an open access article distributed under the terms and conditions of the Creative Commons Attribution (CC-BY) license (http://creativecommons.org/licenses/by/4.0/). 